\title{
Partially Linearized Algorithms in Gyrokinetic Particle Simulation
}

\author{
A. M. Dimits" and W. W. Lee \\ Princeton Plasma Physics Laboratory, Princeton University \\ Princeton, New Jersey 08543
}

In this paper, particle simulation algorithms with time-varying weights for the gyrokinetic Vlasov-Poisson system have been developed. The primary purpose is to use them for the removal of the selected nonlinearities in the simulation of gradient-driven microturbulence so that the relative importance of the various nonlinear effects can be assessed. It is hoped that the use of these procedures will result in a better understanding of the transport mechanisms and scaling in tokamaks. Another application of these algorithms is for the improvement of the numerical properties of the simulation plasma. For instance, implementations of such algorithms 1) enable us to suppress the intrinsic numerical noise in the simulation, and 2) also make it possible to regulate the weights of the fast-moving particles and, in tum, to eliminate the associated high frequency oscillations. Examples of their application to drift-type instabilities in slab geometry are given. We note that the work reported here represents the first successful use of the weighted algorithms in particle codes for the nonlinear simulation of plasmas.

- Present Address: Lawrence Livermore National Laboratory, Livermore, California 94550 


\section{Introduction}

In this paper, we develop and apply partially linearized particle simulation algorithms, in which the particles have time-varying weights, to the following problems in the gyrokinetic simulation of plasma microinstabilities:

1. the removal of the selected nonlinearities in the simulation of drift waves in order to assess the relative importance of these nonlinearities,

2. the suppression of high frequency oscillations in these simulations by regulating the weights of the fast-moving particles so as to improve the numerical properties of the simulation plasma, and

3. the reduction of intrinsic numerical noise due to the free-streaming motion of the simulation particles.

For the past two decades, particle simulation has been an esseritial tool for the investigation of nonlinear kinetic phenomena in plasmas in the areas of fusion, space and particle-beam physics [13]. Standard particle codes use particles with constant weights to solve the Klimontovich equation, which is in the continuity form with no sources, by following the trajectories of the particles under the influence of the self-consistent fields. There is considerably less experience with the other possible nonlinear kinetic simulation approach, namely, the so-called Vlasov code simulation method [4], in which the Vlasov equation is solved numerically using the method of characteristics. Difficulties with this approach associated with need for a velocity space grid have prevented its wider application. These difficulties are 1) the greater computational resources required than for particle simulation, especially for multi-dimensional simulations, and 2) accuracy problems associated with the phase-space filamentation.

However, Vlasov codes have several distinct advantages over particle codes. For example, one can easily turn on and off the various nonlinear terms in the governing equation to assess their relative importance. Furthermore, one can also assign arbitrarily low levels of the initial perturbations to give a better assessment of the linear growth or damping of the waves. (This is especially useful for investigating marginally stable phenomena for plasmas near equilibrium.) These special features were indeed used in a mode-coupling Vlasov code to determine the mechanisms for the saturation and transport for drift instabilities $[5,6]$. The code was written after the observation had been made, in the $2-1 / 2$ dimensional gyrokinetic particle simulation of drift instabiisties, that the resulting fluctuation spectrum was dominated by only a few unstable modes.

It is not, however, practical to expand this Vlasov code to include more unstable modes, nor is possible to incorporate these features into the usual particle codes in which constantweight particles are used. These difficulties prompted us to develop algorithms with variable particle weights for gyrokinetic particle simulation [7]. The time dependence of the weights is a consequence of the source terms that appear in the gyrokin tic Vlasov (or Klimontovich) equation when the parallel acceleration and/or the background inhomogeneity effects (but not the nonlinear $\boldsymbol{E} \times \boldsymbol{B}$ convection) are linearized. So far, we have used the partially linearized schemes in $2-1 / 2$ dimensional simulations to determine whether the nonlinear parallel acceleration plays any role in the observed nonlinear saturation and particle transport, and whether the asymmetry in $E \times B$ trapping observed in simulations that use the "multi-scale model" is indeed physical [8,9]. In time, these algorithms will prove to be useful for microturbulence studies in three dimensions. 
One important application of the present partially-linearized algorithms is their use to improve the numerical properties of the simulation plasma such as time step, grid spacing and noise level. For example, by assigning the prescribed weights of $e \phi(x) / T_{e}$ to the fast moving electrons and thereby forcing upon them the Boltzmann-like response, we can eliminate the undesirable high frequency oscillations from the simulation. Thus, for this instance, ion accoustic waves (and the associated drift-type waves) become the only normal modes in the system resulting in a considerable improvement in the numerical properties. There are, however, complications which we will discuss later. The issue of excessive numerical noise due to the use of the limited number of particles in the simulation has been an area of concem in recent years [10]. For example, the noise level can sometimes be high enough that the physical instability of interest is suppressed [10]. This is especially true when the instability is weak. With the present algorithms, the initial intrinsic noise can be totally eliminated by simply zeroing out the contributions from the zero-order part of the distribution function for a spatially homogeneous plasma. (The additional requirement that the particle motion is incompressible in the configuration space is usually satisfied, except when the "multi-scale model," is used, as we will elaborate later.) This is a straightforward extension of the noise suppression techniques [11] used for the fully linearized particle simulation schemes [12]. Recently, a noise reduction scheme for fully nonlinear particle simluation has also been investigated by Kotchenreuther [13]. We will remark on this later.

There are two basic particle simulation methods for solving partially inearized systems. In one method, due to Freidberg et al. [12] (see also Cohen et al. [14]), the Klimontovich distribution function is expanded to the first order with respect to the partially linearized zero-order particle trajecturies in the phase space. The appropriate equations of motion for the perturbed phase space quantities are then obtained. The resulting distribution is an exact solution of the partially linearized Klimontovich equation. In the second method, which we develop here, the terms containing the zero-order gradients of the distribution function are treated as sources for the partially linearized Klimontovich equation. This results in an assignment of time-varying weights to the particles when it is solved by the particle pushing scheme.

The organization of the paper is as follows. In Sec. 2, the gyrokinetic formalism for the Vlasov-Poisson system and the gyrokinetic particle simulation techniques for drift waves are briefly reviewed. Section 3 presents the extension of the standard linearization model [12] to the partially linearized system. The partially linearized particle simulation algorithms with time-varying weights are described in Sec. 4. The equations for the particular applications to gyrokinetic simulation are given in Sec. 5. A discussion of the phenomena observed in the original gyrokinetic particle simulation of drift waves and their comparisons with the results from the partially linearized weighted-particle algorithms are presented in Sec. 6. In Sec. 7, the suppression of high frequency oscillations and the noise reduction issues are discussed. The summary and the concluding remarks are included in Sec. 8.

\section{Gyrokinetic Particle Simulation and Applications to Drift Waves}

Let us briefly review the gyrokinetic formalism and technique as applied to drift wave simulations. The gyrokinetic particle simulation scheme uses the stanciard particle simulation methods to solve the gyrokinetic systems of equations which are based on the well-known gyrokinetic 
ordering,

$$
\frac{q_{\alpha} \phi}{T_{\alpha}} \sim \frac{\omega}{\Omega_{\alpha}} \sim \frac{\rho_{\alpha}}{L} \sim \epsilon \ll 1, \quad L \sim L_{\|},
$$

where $\rho_{\alpha} \equiv v_{t \alpha} / \Omega_{\alpha}, \Omega_{\alpha} \equiv q_{\alpha} B / m_{\alpha} c, v_{t \alpha} \equiv \sqrt{T_{\alpha} / m_{\alpha}}, q_{\alpha}, m_{\alpha}$, and $T_{\alpha}$ are respectively the charge, mass, and temperature for species $\alpha, c$ is the speed of light, $B$ is the magnetic field strength, $\phi$ is the electrostatic potential, $\omega$ is the frequency of the perturbation, $L$ is a characteristic perpendicular equilibrium scale length of the system, and $L_{\|}$is the characteristic parallel wavelength of the perturbation. We will drop the species index $\alpha$ when we are referring to either species and there is no ambiguity.

The electrostatic gyrokinetic Vlasov equation for a plasma in a uniform magnetic field is $[10,15,16]$

$$
\frac{\partial F}{\partial t}+v_{\|} \hat{\boldsymbol{b}} \cdot \frac{\partial F}{\partial \boldsymbol{R}}-\frac{c}{B} \frac{\partial}{\partial \boldsymbol{R}} \cdot\left[\left(\frac{\partial \Psi}{\partial \boldsymbol{R}} \times \hat{\boldsymbol{b}}\right) F\right]-\frac{q}{m} \frac{\partial \Psi}{\partial \boldsymbol{R}} \cdot \hat{\boldsymbol{b}} \frac{\partial F}{\partial v_{\|}}=C(F)
$$

where

$$
\begin{aligned}
\Psi(\boldsymbol{R}) & \equiv \bar{\phi}-\frac{q}{2 T}\left(\frac{v_{t}}{\Omega}\right)^{2}\left|\frac{\partial \phi}{\partial \boldsymbol{R}}\right|^{2}, \\
\bar{\phi} & \equiv \sum_{\boldsymbol{k}} \phi(\boldsymbol{k}) J_{0}\left(\frac{k_{\perp} v_{\perp}}{\Omega}\right) \exp (i \boldsymbol{k} \cdot \boldsymbol{R}), \\
\boldsymbol{R} & \equiv \boldsymbol{x}+\frac{\boldsymbol{v}_{\perp} \times \hat{b}}{\Omega},
\end{aligned}
$$

$\boldsymbol{x}$ is the particle position, $v_{\perp}$ is the perpendicular velocity, $\bar{\phi}(\boldsymbol{R})$ is the gyro-averaged electrostatic potential, $F\left(\boldsymbol{R}, \mu, v_{\|}, t\right)$ is the full gyro-averaged distribution function, $\mu \equiv v_{\perp}^{2} / 2$, and $C(F)$ is the collision operator. The electrostatic potential $\phi$ is given by the gyrokinetic Poisson equation which, for a single ion species $i$, is

$$
\nabla^{2} \phi-\frac{\tau(\phi-\tilde{\phi})}{\lambda_{D}^{2}}+\left(\frac{\rho_{\mathrm{s}}}{\lambda_{\mathrm{D}}}\right)^{2} \nabla_{\perp} \cdot\left[\frac{\left(n^{\mathrm{i}}-n_{0}\right)}{n_{0}} \nabla_{\perp} \phi\right]=-4 \pi e\left(\bar{n}^{\mathrm{i}}-n^{\mathrm{e}}\right)
$$

where

$$
\begin{aligned}
& \tilde{\phi}(\boldsymbol{x}) \equiv \sum_{\boldsymbol{k}} \phi(\boldsymbol{k}) \Gamma_{0}\left(k_{\perp}^{2} \rho_{\mathrm{i}}^{2}\right) \exp (i \boldsymbol{k} \cdot \boldsymbol{x}) \\
& \bar{n}(\boldsymbol{x}) \equiv \sum_{\boldsymbol{k}} \int F(\boldsymbol{k}) J_{0}\left(\frac{k_{\perp} v_{\perp}}{\Omega}\right) \exp (i \boldsymbol{k} \cdot \boldsymbol{R}) d \mu d v_{\|}
\end{aligned}
$$

and where $\tau \equiv T_{\mathrm{e}} / T_{\mathrm{i}}, \rho_{\mathrm{s}} \equiv c_{\mathrm{s}} / \Omega_{\mathrm{i}}, c_{\mathrm{s}} \equiv \sqrt{T_{\mathrm{e}} / m_{\mathrm{i}}}, k_{\perp}$ is the perpendicular wave number, $\lambda_{\mathrm{D}} \equiv \sqrt{T_{e} / 4 \pi n_{0} e^{2}}$ is the electron Debye length, $n_{0}$ is the background ion number density, $n^{\alpha}$ is the number density of species $\alpha$, and $\Gamma_{0}(b) \equiv I_{0}(b) e^{-b}$. For ions, $C(F)=0$. For the electrons, finite gyroradius effects are negligible so that $\Psi=\bar{\phi}=\widetilde{\phi}=\phi$. Pitch-angle scattering is retained for the electrons, via a Monte-Carlo model for the Lorentz cillision operator [6],

$$
C\left(F_{\mathrm{e}}\right)=\frac{\nu_{\mathrm{ei}}}{2 \sin \xi} \frac{\partial}{\partial \xi}\left(\sin \xi \frac{\partial F_{e}}{\partial \xi}\right) .
$$


Here $\nu_{e i}$ is the electron pitch-angle scattering frequency, which in these studies is taken to be velocity-independent, and $\xi$ is the pitch angle. Since the parailel phase velocity of the waves is nsually smaller than the electron thermal velocity, the population of runaway electrons should be negligible. The intrinsic collision time due to the particle discreteness in the simulation is several orders of magnitude greater than the typical run time [6].

With the gyrokinetic system, there is no longer any need to follow the details of the gyromotion. The resulting relaxed time step and grid size requirements [10] make tractable the simulation of low-frequency $\left(\omega \ll \Omega_{\mathrm{i}}\right.$ ) microinstabilities well into the nonlinearly saturated regime $[5,6,15,17,18]$ and represent an important advance in the path towards understanding the nonlinear evolution of these microinstabilities.

To model a driven system due to the preserce of spatial gradients in a periodic geometry, we use a simple particle conserving generalization of the standard source term for the gradients commonly used in linear theory [19]. The perpendicular configuration-space velocity in Eq. (1a), is modified so that it becomes [10]

$$
v_{\text {perp }} \equiv \frac{d \boldsymbol{R}}{d t}=-\frac{c}{B}\left[\frac{\partial \Psi}{\partial \boldsymbol{R}}+\kappa \Psi\right] \times \hat{b}
$$

where $\kappa \equiv-\nabla \ln F_{M}=\kappa \hat{\boldsymbol{x}}$ and $\hat{b} \equiv \hat{\boldsymbol{z}}+\theta \hat{\boldsymbol{y}}$. Here, $F_{M}$ is the background Maxwellian distribution function with the associated density $n_{0}(x)$ and temperature $T_{0}(x)$. We can write $\kappa$ as $\kappa=$ $\kappa_{n}\left[1+\eta\left(v_{\|}^{2} / v_{t \alpha}^{2}-\frac{1}{2}\right)\right]$, where $\eta \equiv\left(d \ln T_{0} / d \ln n_{0}\right)$ and $\kappa_{n}$ represents the density gradient. For $\eta=0, \kappa=\kappa_{n}$.

This velocity has the important property

$$
\boldsymbol{\nabla} \cdot \boldsymbol{v}_{\text {perp }}=-\kappa \dot{X},
$$

where $\dot{X}=-(c / B)(\partial \Psi / \partial Y)$ is the $x$ component of $v_{\text {perp. }}$ This results in the addition of the term

$$
\frac{c}{B} \frac{\partial}{\partial \boldsymbol{R}} \cdot[\hat{b} \times \kappa(\psi F)]
$$

to the left hand side of Eq. (1a).

\section{Extension of the Standard Linearization Method}

A linearized particle simulation algorithm was developed by Freidberg pr al. [12] in 1969, and has been used for the calculation of (fully-) linear stability properties of plasma equilibria for which analytical methods are unavailable $[11,12,20]$. This algorithm can be applied to the partial linearization of selected terms as follows.

The general form of the kinetic equation solved by a standard particle simulation code is that of a continuity equation for the Klimontovich density:

$$
\frac{\partial f}{\partial t}+\partial_{i}\left(V_{i} f\right)=0
$$

where the repeated indices denote summation over all phase-space components,

$$
f(X, t)=\sum_{j=1}^{N} \delta^{D}\left(X-X_{j}(t)\right),
$$


$j$ is the particle index, $N$ is the particle number, $D$ is the dimensionality of the single particle phase space, $\mathrm{X}$ is the phase-space coordinate vector, and $V \doteq d X / d t$. It is then generally argued that, provided that the initial conditions are chosen properly and the fields are calculated in such a way as to render the simulation collisionless, a single realization of the solution of Eq. (2) should suitably represent the solution for the Vlasov equation that is formally the same as Eq. (2).

Suppose now that

$$
\begin{aligned}
& V(X, t)=V^{(0)}+\epsilon V^{(1)}, \\
& f(X, t)=f^{(0)}+\epsilon f^{(1)},
\end{aligned}
$$

where $\epsilon$ is a small parameter, and we wish to solve Eq. (2) correct to first order in $\epsilon$. The resulting perturbed kinetic equations are

$$
\begin{aligned}
& \frac{\partial f^{(0)}}{\partial t}+\partial_{i}\left(V_{i}^{(0)} f^{(0)}\right)=0 \\
& \frac{\partial f^{(1)}}{\partial t}+\partial_{i}\left(V_{i}^{(0)} f^{(1)}\right)=-\partial_{i}\left(V_{i}^{(1)} f^{(0)}\right),
\end{aligned}
$$

where the index $i$ is a phase space component index. Their solution is

$$
\begin{aligned}
f^{(0)} & =\sum_{j=1}^{N} \delta^{D}\left(X-X_{j}^{(0)}(t)\right), \\
f^{(1)} & =-\sum_{j} X_{j, i}^{(1)}(t) \partial_{i} \delta^{D}\left(X-X_{j}^{(0)}(t)\right),
\end{aligned}
$$

where $X_{j, i}$ denotes the $i$-th component of the phase-space-position vector for the $j$-th particle, and where $X^{(0)}$ and $X^{(1)}$ satisfy the perturbative equations of motion,

$$
\begin{aligned}
& \dot{X}^{(0)}(t)=V^{(0)}\left(X^{(0)}(t), t\right), \\
& \dot{X}^{(1)}(t)=X_{i}^{(1)} \hat{\theta}_{i} V^{(0)}\left(X^{(0)}(t), t\right)+V^{(1)}\left(X^{(0)}(t), t\right) .
\end{aligned}
$$

The first term on the right-hand side of Eq. (3d) is just the first-order term of the Taylor expansion of $V^{(0)}(X(t), t)$ about $X=X^{(0)}(t)$. The electromagnetic fields are then calculated from the total density, which comes from the combined distribution function $f^{(0)}+f^{(1)}$. In the case of full linearization, $f^{(0)}$ does not contribute to the non-equilibrium density perturbation so the density can be evaluated from $f^{(1)}$ alone. This gets rid of the equilibrium noise [11]. Each simulation particle in this algorithm contributes, in general, a different amount to the particle density. In this sense, the particles can be regarded as "weighted". In the linearization method presented in the next section, the particles are also weighted. There, however, the weights appear as numerical factors rather than as differential operators acting on the $\delta$ functions. The issue of ignoring the equilibrium noise for the partially linearized algorithms will be discussed later.

The random velocity $\delta V_{c}$ that models collisions, if any, is most conveniently retained as a part of $V^{(0)}$. It is straightforward to show that the collisions are correctly treated in this way. Collision models such as those of Shanny, Dawson, and Greene [21], and of Boozer and KuoPetravic [22] consist of adding a random parallel velocity, constructed to have the same statistical properties as $v_{\|}$in real pitch-angle scattering. In any set of variables, the pitch-angle collision 
operator consists of a generalized diffusion term and a (possibly zero) drag term. As long as the collisionless characteristics remain smooth, when discretized after linearization, the linearization does not change the diffusion term. The drag term is even more robust under linearization.

Fully linearized codes are of particular utility for the study of the linear stability in geometries that have one or more symmetry direction. Fourier analysis in symmetry directions can then be used to reduce the dimensionality of the simulation $[11,12]$. In the partially linearized case, where there are still nonlinear terms present, such a simplification is not possible.

We have implemented this method for the removal of the parallel nonlinearity in the gyrokinetic simulation of drift waves. As they stand, Eqs. (3c) and (3d) are unsuitable for situations where the zero-order motion is near-periodic [8], as in the states observed in the gyrokinetic simulations of drift waves $[5,6,8]$. The difficulty is that for deeply $E \times B$-trapped particles, the matrix $\partial_{i} V^{(0)}$ in Eq. (3d) has eigenvalues at the $\boldsymbol{E} \times \boldsymbol{B}$-trapping frequency. The beating between the periodic source term [the $V^{(1)}$ term in Eq. (3d)] with these eigenfrequencies causes a secular growth of $X^{(1)}$ and $Y^{(1)}$. Physically, this secularity represents the failure of Eqs. (3) to describe the shifts in the position of the $O$ points and the values of the trapping frequencies due to the parallel acceleration [8]. The effect of this secularity has also been observed in a non-self-consistent electron gyrocenter code run which used this algorithm. The gyrocenter flux was observed to grow very large (many times the physical value), with no identifiable bound. For the small $\theta$ (for example, $\theta=0.002$,) cases of interest for the use of the above algorithm to remove the parallel nonlinearity, these shifts are very small, as was shown in our previous work [8]. Thus, this algorithm can be salvaged by removing the $X_{i}^{(1)} \partial_{i} V^{(0)}$ from Eq. (3d). When this is done, the behavior of the resulting simulations is similar to that of simulations for similar parameters made using the weighted particle method to be described in the subsequent sections. We do not persue this method further here.

\section{Weighteci Particle Method}

In our scheme, the terms to be linearized are regarded as source terms in the solution by the method of characteristics. This results in particle weights which are numerical factors rather than quantities involving derivatives acting on the $\delta$ functions. The fact that the weights are numerical factors, rather than derivative operators, makes this method more versatile in many applications than the standard method.

To derive the algorithm, the terms in the Vlasov equation to be linearized are first approximated by terms of the form $g(X, t) f^{(0)}$, where $g$ is some function of the phase-space coordinates and time, and $f^{(0)}$ is the zero-order distribution function, which solves the Vlasov equation in the absence of the term to be linearized. The justification for the replacement depends on the particular replacement made. For the removal of the parallel nonlinearity, the replacement $\partial f / \partial v_{\|} \rightarrow-v_{\|} f^{(0)} / v_{t}^{2}$ is made in the parallei acceleration term in the gyrokinetic Vlasov equation (1a), so that

$$
g=-\frac{q}{m} E_{\|} \frac{v_{\|}}{v_{\mathrm{t}}^{2}}
$$

This replacement is justified by noting that it is valid if $f^{(0)}$ is a Maxwellian and that $g \delta f$, where $\delta f$ is the departure between $f^{(0)}$ and the initial Maxwellian, is one order smaller in 
the gyrokinetic smallness parameter than $g f^{(0)}$. For the removal of the nonlinear $\kappa$ term, the analogous replacement

$$
\kappa \frac{\partial}{\partial y}(\phi f) \rightarrow \kappa \frac{\partial}{\partial y}\left(\phi f^{(0)}\right) \rightarrow \kappa f^{(0)} \frac{\partial \phi}{\partial y}
$$

is made, so that we have

$$
g=\kappa \frac{\partial \phi}{\partial y}
$$

This is again argued to be valid because the differences between the expressions in Eq. (4) are one order smaller in the gyrokinetic smallness parameter than the last of the expressions. The resulting equation is then of the form

$$
\frac{\partial f}{\partial t}+\partial_{i}\left(V_{i}^{(0)} f\right)=g(X, t) f^{(0)}
$$

This is then rewritten as

$$
\frac{d f}{d t}+\left(\partial_{i} V_{i}^{(0)}\right) f=g(X, t) f^{(0)},
$$

with the characteristics given by

$$
\frac{d X}{d t}=V^{(0)}(X, t)=\frac{d}{d t} X^{(0)}(t) .
$$

The formal solution is

$$
f(X, t)=f\left(X_{0}, 0\right)\left[1+\int_{0}^{t} d t^{\prime} g\left(X^{\prime}, t^{\prime}\right)\right] \exp \left[-\int_{0}^{t} d t^{\prime} \partial_{i}^{\prime} V_{i}^{(0)}\left(X^{\prime}, t^{\prime}\right)\right],
$$

where $X^{\prime} \doteq X^{(0)}\left(t^{\prime}\right)$ and $X_{0} \doteq X^{(0)}(0)$. Using

$$
f^{(0)}(X, t)=f\left(X_{0}, 0\right) \exp \left[-\int_{0}^{t} d t^{\prime} \partial_{i}^{\prime} V_{i}^{(0)}\left(X^{\prime}, t^{\prime}\right)\right]
$$

this can be rewritten as

$$
f(X, t)=f^{(0)}(X, t)\left[1+\int_{0}^{t} d t^{\prime} g\left(X^{\prime}, t^{\prime}\right)\right] .
$$

Applying this to particle simulations, we have

$$
f^{(0)}(X, t)=\sum_{j} \delta^{D}\left(X-X_{j}^{(0)}\right)
$$

so that

$$
\begin{aligned}
f & =f^{(0)}+f^{(1)} \\
& =\sum_{j} \delta^{D}\left(X-X_{j}^{(0)}(t)\right)\left[1+\int_{0}^{t} d t^{\prime} g\left(X_{j}^{(0)}\left(t^{\prime}\right), t^{\prime}\right)\right] .
\end{aligned}
$$

This formula lends itself directly to particle simulation. The new scheme requires the storage of two additional variables per particle to account for the first-order velocity perturbations and spatial displacements. For our application, this is an increase of less than $30 \%$. The resulting algorithms that were implemented will now be summarized. 


\section{Particular Applications of the Weighted Particle Method}

\subsection{Linearized- $E_{\|}$Algorithm}

The gyrokinetic Vlasov equations, partially linearized with respect to the parallel acceleration term, are

$$
\begin{aligned}
\frac{\partial f^{(1)}}{\partial t}+\nabla_{\perp} \cdot\left\{\left[v_{E \times B}+\left(\kappa \Psi+\theta v_{\|}\right) \hat{\boldsymbol{y}}\right] f^{(1)}\right\}-\frac{q}{m} \frac{v_{\|}}{v_{t}^{2}} E_{\|} f^{(0)} & =C\left(f^{(1)}\right) \\
\frac{\partial f^{(0)}}{\partial t}+\nabla_{\perp} \cdot\left\{\left[\boldsymbol{v}_{E \times B}+\left(\kappa \Psi+\theta v_{\|}\right) \hat{\boldsymbol{y}}\right] f^{(0)}\right\} & =C\left(f^{(0)}\right)
\end{aligned}
$$

where $v_{E \times B} \doteq(q / m \Omega) \hat{b} \times \nabla_{\perp} \Psi$ and $E_{\|} \doteq-(\partial / \partial z) \Psi$. From Eq. (7), these have the solution of

$$
f=f^{(0)}+f^{(1)}=\sum_{j}\left(1+\frac{v_{\| j}^{(0)} v_{\| j}^{(1)}}{v_{t}^{2}}\right) \delta\left(x_{\perp}-x_{\perp j}^{(0)}\right) \delta\left(v_{\|}-v_{\| j}^{(0)}\right),
$$

where

$$
v_{\| j}^{(1)}=\frac{q}{m} \int_{0}^{t} d t^{\prime} E_{\|}\left(x_{\perp j}^{(0)}\left(t^{\prime}\right), t^{\prime}\right)
$$

$x_{\perp} \doteq(x, y)$, and $x_{j}^{(0)}(t)$ and $v_{\| j}^{(0)}(t)$ are the trajectories obeying the initial conditions evolving with zero parallel acceleration.

\subsection{Linearized- $E_{\|} \& \kappa$ Algorithm}

The removal of the multiple-scale model nonlinearity is of interest to determine its effect. Motivated by the agreement, which will be described later, between the results from the linearized$E_{\|}$code of the previous subsection and the full gyrokinetic code, we remove both the parallel and multiple-scale nonlinearities. The linearization is then rigorous in the sense that a Maxwellian is a solution of the zero-order Vlasov equation. The resulting equation is

$$
\begin{aligned}
\frac{\partial f^{(1)}}{\partial t}+\nabla_{\perp} \cdot\left[\left(v_{E \times B}+\theta v_{\|} \hat{\boldsymbol{y}}\right) f^{(1)}\right]+\kappa \frac{\partial \Psi}{\partial y} F_{M}-\frac{q}{m} E_{\|} \frac{v_{\|}}{v_{t}^{2}} F_{M} & \left.=C(\lrcorner^{\prime \prime)}\right) \\
\frac{\partial f^{(0)}}{\partial t}+\nabla_{\perp} \cdot\left\{\left[v_{E \times B}+\theta v_{\|} \hat{\boldsymbol{y}}\right] f^{(0)}\right\} & =C\left(f^{(0)}\right) .
\end{aligned}
$$

The initial Vlasov distribution function has been assumed to be a spatially uniform Maxwellian, in which case it remains one for all time. The corresponding Klimontovich distribution function is

$$
f=f^{(0)}+f^{(1)}=\sum_{j}\left(1+\kappa \Delta x_{j}^{(0)}+\frac{v_{\| j}^{(0)} v_{\| j}^{(1)}}{v_{t}^{2}}\right) \delta\left(x_{\perp}-x_{\perp j}^{(0)}\right) \delta\left(v_{\|}-v_{\| j}^{(0)}\right),
$$

where the zero-order trajectories have neither the parallel acceleration nor the $\kappa$ term. This algorithm is particle conserving as can be established most easily by noting that the integral 
of the first-order Vlasov equation over position and parallel velocity gives $(d / d t) N^{(1)}(t)=0$, where $N^{(1)}(t)$ is the first-order contribution to the particle density.

Note that $\Delta x_{j}^{(0)}$ should be taken to be continuous in time in Eq. (9) rather than periodic in space since this avoids the formation of a discontinuity of the density at the $x$ boundaries of the simulation box. For simplicity, the equations in this section are written in $\left(x, y, v_{\|}\right)$. They can be easily generalized to $\left(x, y, z, \mu, v_{\|}\right)$.

\section{Drift Wave Simulation with Partially Linearized Algorithms}

The geometry used in the gyrokinetic drift wave simulations is a slab with one ignorable position coordinate, $z$. The magnetic field is uniform and inclined to the $z$ axis, $B=B(\hat{z}+\theta \hat{y})$, where $\hat{z}$ and $\hat{y}$ are the unit vectors in the $z$ and $y$ directions. The parallel wavenumber is then $k_{\|}=\theta k_{y}$. The inclination angle $\theta(\ll 1)$ is a constant in the shear-free slab models. As defined above, the background gradient (for our multi-scale model) is in the $x$ direction. Since this is a shear-free system, we can assume that both the particles and waves are periodic in both $x$ and $y$. The simulation parameters are: $L_{x} \times L_{y}=32 \Delta \times 32 \Delta$, where $\Delta$ is the grid size, $N$ (the number of simulation particles per species) $=128 \times 128, m_{\mathrm{i}} / m_{\mathrm{e}}=1837, \tau=T_{\mathrm{e}} / T_{1}=4$ or 100 , particle size $=2 \Delta, \theta=0.002$ or $0.01, \rho_{\mathrm{s}}=4.286 \Delta, \kappa \rho_{\mathrm{s}}=0.214,\left(k_{x} \rho_{\mathrm{s}}, k_{y} \rho_{\mathrm{s}}\right)=(0.842 \mathrm{~m}, 0.842 n)$, $\omega_{m} / \Omega_{\mathrm{i}}=0.18 n$, where $(m, n)=0, \pm 1, \pm 2, \ldots, \nu_{\text {ei }} / \Omega_{\mathrm{i}}=0$ or $0.001, \Omega_{\mathrm{i}} \Delta t=1.09,2.18$, or 5.45 , and $\Delta t$ is the time step used in the code. The $k_{x}=0$ electrostatic potentials are zeroed out in the code, i.e., $\phi(m=0, n \neq 0)=0$, to satisfy the requirement of $\left|k_{x}\right| \gg|\kappa|$ for the multi-scale model [10]. For simplicity, we have also set $\phi(m \neq 0, n=0)=0[5,6]$.

Following the convention used in the papers of Lee and coworkers, in most of what follows, the system of units used has lengths scaled to $\rho_{s}$, times scaled to $\Omega_{i}^{-1}$, densities scaled to the mean density $n_{0}$, and electrostatic potentials scaled to $T_{e} / e$, where $e$ is the unsigned charge of the electrons.

Figure 1 shows the time histories of the amplitudes of the $(m=1, n= \pm 1)$ modes of the electrostatic potential, denoted by $\phi_{ \pm}$, for a typical semi-coherent gyrokinetic drift wave run with $\theta=0.002, \tau=100, \nu_{\mathrm{ei}}=0.001$, and $\Delta t=1.09$. They are the dominant fluctuations in the simulation and the linear theory indicates that they also correspond to the most unstable modes in the system with $\omega= \pm 0.034+0.027 i[5,6]$. The measured values in the linear stage of the development are in good agreement with the theory and the other phenomena observed can be summarized as follows:[5,6,8]

1. The time evolution of the instability can be divided into pre-normal-mode, linear, saturation, and steady-state phases.

2. In addition to $\phi, n^{e}$, and $\bar{n}^{\mathrm{i}}$ associated with the $(1, \pm 1)$ modes, nonlinearly generated $(2,0)$ and $(0,2)$ mode fluctuations in the density have also been observed.

3. The + and - modes oscillate with the nonlinearly modified real frequencies in the post-linear phases. The nonlinear modifications include:

- a upward frequency shift to $\omega_{r} \simeq \pm 0.055$,

- a frequency broadening. 
- a frequency difference between + and - modes implying a net $x$ component of the phase velocity, $\omega / k_{x}$, and

- amplitude fluctuations (around a mean value of ' $7 \%$ ) due to an energy exchange between the + and - modes.

4. A steady-state particle flux, due almost entirely to the + and - modes, has a value much less than that from the quasilinear theory. It increases with $\nu_{\mathrm{ei}}$ and decreases with $\theta$.

We have carried out the drift wave simulation based on the linearized- $E_{\|}$algorithm. (In the actual coding, both the ion and electron parallel nonlinearities were removed, although its effect on the ion motion is insignificant in this case.) Figures 2 and 3 show the time histories for the electrostatic potentials and the resulting electron particle flux, respectively, where the latter is calculated using[10]

$$
\left\langle\Gamma_{e x}\right\rangle=\frac{1}{N} \sum_{j=1}^{N}\left(1+w_{j}\right) \boldsymbol{V}_{E \times B \cdot \hat{x}},
$$

where

$$
\boldsymbol{V}_{E \times B} \equiv-\frac{c}{B} \frac{\partial \Psi}{\partial \boldsymbol{R}} \times \hat{\boldsymbol{b}}
$$

and $w_{j}$ is the contribution from the linearizaton. Since the parameters used are identical to those of Fig. 1, there is evidently a satisfactory qualitative (although not quantitative) agreement between the two runs. For instance, the linearized- $E_{\|}$run saturates at a slightly higher level of $9 \%$ and also oscillates at somewhat higher frequencies of $\omega_{r} \simeq \pm 0.067$. In the "steady-state" phase of the evolution, the two runs have similar values of the particle flux as well as for the frequency difference ( $x$ phase velocity) (Fig. 3 should be compared with Fig. 4 of Ref. 9). However, the level of the energy exchange between the + and - modes is reduced in the linearized- $E_{\|}$run.

Thus, althougt there are quantitative differences between the results from the partially linearized code and the original code, they indicate that the same saturation mechanisms are operative. This is consistent with the picture of the saturation mechanism portrayed in our previous work $[8,9]$, namely, the increased real frequencies in the nonlinear stage necessitate higher saturation levels so that the ion nonlinearity can come into play to maintain the ambipolarity of the particle diffusion.

To demonstrate the physical mechanism for the reduction of the electron flux by the parallel acceleration in the nonlinear stage of the simulation shown in Fig. 3, we have used a non-selfconsistent implementation of Eqs. (8a-d). The code follows the motion of gyrocenters and uses the electrostatic potentials $\phi_{ \pm}$with similar time evolution and spatial structure as those shown in Fig. 1. (Thus, the code is the same as the linearized- $E_{\|}$code except that the calculation of the selfconsistent potentials from the gyrokinetic Poisson equation is omitted.) As seen from Fig. 4, this non-self-consistent code successfully models the flux reduction. The underlying mechanism is the depopulation of the parallel resonance, represented by a decrease in the weights of the particles as they diffuse radially. This decrease in the weights of the diffusing electron gyrocenters causes a decrease in the weighted flux as well as in the total (weighted) number of the electrons at the resonance, as shown in Fig. 5. 
We now discuss the effect on the nonlinear parallel acceleration term for the various simulation parameters. For $\nu_{\mathrm{ej}}=0, \tau=100, \theta=0.002$, the agreement between the linearized- $E_{\|}$ run and the fully nonlinear run is even closer than for $\nu_{\mathrm{ei}}=0.001$, both quantitatively and qualitatively. However, for $\tau=4, \nu_{\text {ei }}=0.001$, and $\theta=0.002$, the increase in the saturation levels and the upward frequency shifts in the linearized- $E_{\|}$code are somewhat greater than for $\tau=100$. For larger $\theta$, we expect the differences between the full code and the linearized- $E_{\|}$. code to be more substantial, and this is bome out by the simulation results. The results are shown for a fully nonlinear run in Fig. 6, and a linearized- $E_{\|}$run in Fig. 7, respectively, for $\tau=4, \nu_{\text {oi }}=0, \theta=0.01$, and $\Delta t=2.18$. The linear theory predicts $\omega= \pm 0.06+0.011 i$. As we can see, the linear growth is obscured by the noise (although it can be clearly seen when the signal is filtered), especially for the fully nonlinear run. The saturation level of the potential in Fig. 6 is on the average less than $1 \%$ with $\omega_{r}= \pm 0.045$, while in Fig. 7 we have $e \phi / T_{\mathbf{c}}=5 \%$ and $\omega_{\mathrm{r}}= \pm 0.04$. Thus, we indeed see that parallel acceleration nonlinearity, i.e., the nonlinear- $E_{\|}$term, becomes important when $k_{\|}$is large, in agreement with the usual understanding. These results serve as an example the usefulness of the linearized- $E_{\|}$algorithm for understanding the nature of microturbulence. One important aspect of Fig. 6 is that the steady state fluctuation level of the instability is nearly the same as the intrinsic numerical noise as given by $\left|e \phi / T_{\mathrm{e}}\right| \simeq 1 /\left(\sqrt{N} k_{\perp} \rho_{\mathrm{s}}\right) \simeq 0.66 \%$, where $N$ is the total number of simulation particles [10]. The question of whether the noise interferes with the instability will be discussed in the next section, along with schemes to reduce the noise.

Simulation runs using the linearized- $E_{\|} \& \kappa$ code have also been carried out. For such a code, the only nonlinearity comes from the $E \times B$ advection and, as such, it is the same model as the one on which most of the current understanding has been based. For the same parameters as those of Figs. 6 and 7, the results indicate that after the linear growth ceases, there is a continued secular growth for at least one of the modes (either + or - ) with an amplitude going beyond $e \phi / T_{e} \simeq 10 \%$. The same behavior is also observed for $\theta=0.002$. The reason for this secular growth can also be understood from the picture given in our previous work [8,9]. In the algorithm specified by Eq. (9), the weight of a particle can increase or decrease linearly without bound as it moves in the $x$ direction. Thus in the semi-coherent saturated states in which the waves have a nonlinear radial propagation, the dominant modes of the density can grow without bound as the $\boldsymbol{E} \times \boldsymbol{B}$-trapped groups of particles are advected. Physically, this growth represents the arrival of $\boldsymbol{E} \times \boldsymbol{B}$-trapped groups of particles which originated in regions of ever higher and higher density. Such a system represents a density profile in which the density runs all the way from $-\infty$ to $+\infty$. The nonlinear driving term in the multi-scale model limits the secular growth of the dominant modes of the densities and potentials due to coherent radial advection, effectively mimicking upper and lower bounds on the densities contained in the equilibrium density profile. Another observation is that energy exchange between the two unstable modes is reduced indicating that the nonlinear $E_{\|}$and $\kappa$ effects, along with the ion $E \times B$ nonlinearity are responsible for the exchange. The zero-order particle flux should remain zero in these runs, because the initial distribution of the simulation plasma is a spatially uniform Maxwellian and $\phi(k=0)=0$. Any deviation from zero is a measure of either numerical or statistical error in the code.

Preliminary investigations involving more turbulent simulations (the detailed results of which will be presented in a future publication), in which there are many unstable modes, indicate that the removal of the nunlinear $\kappa$ term has little effect in turbulent situations. In fact, the saturation level of the modes and of the resulting flux are essentially the same as those of the 
corresponding run using the fully nonlinear code. Thus, we may conclude that the nonlinear part of the driving term of the multiple-scale model has little effect if the saturated states are turbulent (spatially incoherent) with characteristic radial scales shorter than the mean gradient scale lengths. Comparisons between the simulations based on the nonlinear- and linear- $\kappa$ terms will be discussed further in the next section.

\section{Jong Time Step and Noise Suppression using Time-Varying Weights}

This section describes a detailed study on the use of the time-varying weights in the partially linearized algorithms for the purpose of increasing the time step and grid spacing, and decreasing the noise level in the simulation. Let us first start with the equation for the weights due to the linearization of the parallel electric field. From Sec. 5.1, we have

$$
\frac{d w_{j}}{d t}=\frac{q}{m}\left(\frac{v_{\| j}^{(0)}}{v_{\mathfrak{t}}^{2}}\right) E_{\|}\left(\boldsymbol{x}_{j}^{(0)}\right)
$$

where $w_{j} \equiv v_{\| j}^{(1)} v_{\| j}^{(0)} / v_{t}^{2}$ and $d / d t=\partial / \partial t+v_{\|} \partial / \partial x_{\|}$. For the fast moving particles in response to the slow waves, we have $\partial / \partial t \ll v_{\|} \partial / \partial x$, i.e., $\omega \ll k_{\|} v_{\|}$. Here, we also assume $\left|V_{E \times B} \cdot \nabla_{\perp}\right| \ll$ $\left|v_{\|} \partial / \partial x_{\|}\right|$for these particles. Since $E_{\|}=-\partial \Psi / \partial x_{\|}$and $v_{\mathrm{t}}^{2}=T / m$, we obtain for the $\mathrm{j}$-th electron,

$$
w_{j}=e \Psi\left(\boldsymbol{x}_{j}^{(0)}\right) / T_{\mathrm{e}} .
$$

While the zero-order trajectories for the slow particles are still governed by

$$
\frac{d x_{j}^{(0)}}{d t}=V_{E \times B, j}+\kappa \Psi\left(x_{j}^{(0)}\right) \hat{y}+v_{\| j}^{(0)} \hat{b},
$$

and

$$
\frac{d v_{\| j}^{(0)}}{d t}=0
$$

the equations of motion for the fast particles are now given by

$$
\frac{d \boldsymbol{x}_{j}^{(0)}}{d t}=V_{E \times B, j}+v_{\| j}^{(0)} \hat{b},
$$

and Eq. (12b) instead. This is because the adiabatic particles should no longer be under the influence of the external drive. [In Eq. (13), the $\boldsymbol{E} \times \boldsymbol{B}$ term is much smaller than the parallel drift for the fast particles.] The corresponding distribution function for this system of slow and fast particles remains as

$$
f=f^{(0)}+f^{(1)}=\sum_{j=1}^{N}\left(1+w_{j}\right) \delta\left(x-x_{j}^{(0)}\right) \delta\left(v_{\|}-v_{\| j}^{(0)}\right),
$$


where $w_{j}$ 's are given by either Eqs. (10) or (11). For the linearized- $E_{\|} \& \kappa$ case, Eqs. (10), (11), (12b), and (13) are still valid, and Eq. (14) changes to

$$
f=f^{(0)}+f^{(1)}=\sum_{j=1}^{N}\left(1+w_{j}+\kappa \Delta x_{j}^{(0)}\right) \delta\left(x-x_{j}^{(0)}\right) \delta\left(v_{\|}-v_{\| j}^{(0)}\right),
$$

where $\Delta x_{j}^{(0)}$ is given by

$$
\frac{d \Delta x_{j}^{(0)}}{d t}=-\frac{q}{m \Omega} \frac{\partial \Psi\left(\boldsymbol{x}_{j}^{(0)}\right)}{\partial y}
$$

for the slow particles and

$$
\frac{d \Delta x_{j}^{(0)}}{d t}=0
$$

for the fast particles. Again, Eq. (17) indicates that inhomogeneity has no effect on the adiabatic particles.

In fusion and space physics, we are often faced with the situation in which the relevant frequencies satisfy the condition $\left|\omega / k_{||} v_{\text {te }}\right| \ll 1$, where $v_{\text {te }}$ is the thermal velocity of the electrons. Thus, since most of the electrons travel much faster than the phase velocity $\omega_{r} / k_{\|}$of such lowfrequence waves, we can assume that their response is nearly Boltzmann-like, i.e., adiabatic as given by Eq. (11). The perturbed density from Eq. (14) then takes on the well-known form

$$
\delta n^{e}=\int f^{(1)} d v_{\|}=\sum_{j=1}^{N}\left[e \Psi\left(\boldsymbol{x}_{j}^{(0)}\right) / T_{e}\right] \delta\left(\boldsymbol{x}-\boldsymbol{x}_{j}^{(0)}\right)=\left(e \Psi / T_{e}\right) n_{0},
$$

where $n_{0}(x)$ is the zero-order number density and $n_{0}(x)=n_{0}$ for the multi-scale model. This is a very useful approximation when the resonant electron effects are not important. For example, in the simulation of ion temperature gradient drift instabilities[17], such an electron density response has been used in the gyrokinetic Poisson equation, and as a result, there is no need to actually push the electrons in the simulation.

Equation (18), however, totally ignores the nonadiabatic effects of the resonant particles with $v_{\|} \simeq \omega / k_{\|}$, which, through inverse Landau damping, can give rise to a variety of low frequency microinstabilities. The scheme we are about to describe provides a way to recover this important wave-particle interaction. For example, we can use Eq. (11) for the adiabatic electrons with $v_{\| j}^{(0)} \gg \omega / k_{\|}$and apply Eq. (10) for the rest of the electron population to capture the nonadiabatic response. The resulting $w_{j}$ 's for the electrons are then inserted into Eqs. (14) or (15). In the linearized- $E_{\|}$algorithm, the $v_{\| j}^{(0)}$ 's are never updated and there is no need to re-tag the particles. [In the presence of collisions and/or of nonlinear $E_{\|}$, however, $v_{\| j}^{(0)}$ (or $v_{\| j}$ ) will change in time and re-tagging the particles become necessary. This aspect of the scheme is currently under investigation $[23,24]]$.

The obvious numerical advantage of using Eq. (11) is the elimination of the high frequency oscillations, such as the electrostatic shear-Alfven waves, $\omega_{\mathrm{H}} \equiv\left(k_{\|} / k\right) \sqrt{m_{\mathrm{i}} / m_{\mathrm{e}}} \Omega_{\mathrm{i}}$ [10], from the simulation plasma. With the cold hyarodynamic electron response removed $\left(\omega_{\mathrm{r}} / k_{\|} \gg v_{\mathrm{te}}\right)$, these normal modes simply cease to exist. Thus, the time step restriction in the simulation is no longer imposed by the high frequency waves $\left(\omega_{H} \Delta t \lesssim 1\right)$. Instead, it is replaced by the Courant 
condition $k_{\|} v_{\text {te }} \Delta t \lesssim 1$ [10]. Although these two conditions are the same for modes with $k_{\perp} \rho_{\mathbf{s}} \simeq 1$, the numerical implication is totally different. In the absence of the $\omega_{\mathrm{H}}$ modes, we have a unique situation in which fast particles co-exist with slow waves, because $\omega \ll{ }^{\prime} k_{\|} v_{\text {te }}$. One could then use the orbit averaging scheme to effectively increase the time step to $\omega \Delta t \lesssim 1[10,25]$. In the present paper, a simpler alternative will be used. With the $\omega_{\mathrm{H}}$ modes removed, the restriction on grid spacing is also relaxed [10], which makes it possible to simulate a large system with $\Delta x \gg \rho_{s}$.

Another consequence of the elimination of high frequency oscillations is the reduction of the numerical noise (for $k_{\perp \perp} \rho_{\mathrm{s}}<1$ ), because the only normal modes left in the simulation are those associated with the ion acoustic waves [10]. The noise reduction, however, is dependent upon the damping rates of the normal modes, which are usually very small. Thus, one should not expect to actually realize the reduction in most of the simulations, unless artificial dampings are introduced. However, when the particle motion is incompressible in the configuration space, i.e., it obeys Eqs.(12b) and (13), the noise can be reduced, as we will discuss.

To serve as an example for using the algorithm of time-varying weights to increase the time step and to reduce the noise, let us re-visit the simulation runs shown in Figs. 6 and 7, but under somewhat different circumstances. To highlight the similarities between the fully nonlinear simulation and the partially linearized algorithms, we set $\phi(m, n)=\phi^{*}(m,-n)$ in the code. This constraint, which, to a certain degree, mimics a sheared system, has been studied in detail earlier $[5,6,8,9,17]$. In doing so, we eliminate the energy exchange between the + and - modes, which is believed to be mostly caused by the nonlinear velocity space and nonlinear $\kappa$ effects. Consequently, the three-wave coupling process, in which the nonlinear $E \times B$ coupling of the + and - density modes gives rise to the $(2,0)$-mode density perturbation of the electrons (and ions), becomes the dominant nonlinear process in the simulation. The results are given in Fig. 8 . Here, the theoretically predicted linear frequency and growth rate remain the same as before and the nonlinear saturation level is given by [26]

$$
\left|\frac{e \phi}{T_{e}}\right|=\frac{\sqrt{\pi}}{2} \frac{\omega_{\mathrm{r}}\left(\omega_{*}-\omega_{\mathrm{r}}\right)}{k_{\|} \sqrt{m_{\mathrm{i}} / m_{\mathrm{e}}}} \frac{1}{k_{\perp}^{2} \rho_{\mathrm{s}}^{2}\left(1+k_{\perp}^{2} \rho_{\mathrm{s}}^{2} / 2\right)} \simeq 0.72 \%
$$

and $\left|e \phi / T_{e}\right|=\operatorname{Im}\left(\omega / \Omega_{i}\right) /\left(k_{\perp} \rho_{s}\right)^{2}=0.78 \%$ [5], where $\omega_{r}$ is the real frequency. While the measured real frequency $\left(\omega_{r} \simeq 0.06\right)$ and the saturation amplitude $\left(\left|e \phi / T_{e}\right| \simeq 0.6 \%\right)$ agree well with the theoretical predictions, the linear growth is again totally obscured by the noise. This is because the saturation amplitude is nearly the same as the intrinsic noise level of $0.66 \%$ as discussed earlier. The phase velocity of the wave is $v_{\mathrm{ph}}=\omega_{\mathrm{r}} / k_{\|}=0.17 v_{\text {te }}$. Since $\omega_{\mathrm{r}} \ll k_{\|} v_{\text {te }}$, the scheme using regulated weights based on the adaibatic electron response can be utilized to improve the numerical properties of the simulation plasma, i.e., to increase the time step and to reduce the noise.

\subsection{Linearized- $E_{\|}$Model}

One simple way to achieve these goals for the linearized- $E_{\|}$model is to utilize the common approximation that the fast electrons, while responding adiabatically to the waves in a perturbative manner, maintain their zero-order distribution, i.e., $f^{(0)}$ for these particles is unchanged in time. (Thus, for the homogeneous loading, the distribution of the fast particles remains spatially homogeneous.) We can then assume that the trajectories for the particles with $\left|v_{\| j}^{(0)}\right| \gg v_{\mathrm{ph}}\left(\equiv \omega / k_{\|}\right)$ 
obey Eq. (12b) and

$$
\frac{d x_{j}^{(0)}}{d t}=0
$$

where $v_{\text {ph }}$ represents the highest "relevant" phase velocity of interest. In other words, we do not have to push the fast electrons at all, although their adiabatic response is still accounted for. By removing the zero-order free streaming, we get rid of not only the restriction imposed by the $\omega_{\mathrm{H}}$ modes but also the usual Courant condition $k_{\|} v_{\mathrm{te}} \Delta t<1$ when $v_{\mathrm{ph}}<v_{\text {te }}$. Most of all, the dominant source of intrinsic numerical noise, i.e., that associated with the unity term in Eq. (14) for the fast electrons, is now totally suppressed.

Simulation runs using Eqs. (10) and (12) for the ions as well as for the slow electrons with $\left|v_{\| j}^{(0)}\right| \leq 0.3 v_{\text {te }}$, and using Eqs. (11), (19), and (12b) for the fast electrons with $\left|v_{\| j}^{(0)}\right|>0.3 v_{\text {te }}$, where the distribution is given by Eq. (14) and $\Omega_{\mathrm{i}} \Delta t=5.45$, show a distinct linear growth but somewhat lower steady-state fluctuation levels than those of Fig. 8. This is because the background zero-order distribution of the adiabatic particles cannot be accurately represented by the limited number of particles used in the simulation. In this scheme, the contribution to the density from the unity term for the fast particles is nonvanishing but constant in time.

A better way to handle this collisionless case is to use Eqs. (14) and (18) to account for the fast particles and to substitute the results,

$$
\left(n^{e}\right)_{\text {fast }}=\operatorname{erfc}\left(v_{\| \text {cut }}\right)\left(1+e \Psi / T_{e}\right) n_{0},
$$

directly into the gyrokinetic Poisson equation, where erfc is the complimented error function and $v_{\| \text {cut }}=0.3 v_{\text {te }}$ is the cutoff velocity. We, therefore, need only to populate the phase space with the slow electrons and the ions, and to push only these particles in the code. A substantial savings in the computer resources can then be realized. The time history for such a case is given in Fig. 9. Obviously, the noise level is drastically reduced in comparison with the fully nonlinear results in Fig. 8, but the overall behavior is very similar. The initial noise in this case mainly comes from the free streaming motion of the electrons below $v_{\| \text {cut }}$, and the ion contribution is small. The linear stage is clearly visible over two orders of magnitude in the growth of the instability. The saturation amplitude at $0.8 \%$ is also very close to the theoretical value. The frequency and the growth rate are measured as $\omega_{\mathrm{r}}+i \gamma=0.04+0.008 i$, which are somewhat below the linear predictions. The differences are possibly due to the discrepancy between the normal mode approach and the initial value problem solved by the simulation, as well as nonlinear effects just prior to saturation. One more interesting aspect of the simulation in Fig. 9 is the time step. At $\Delta t=5.45$, it satisfies the conditions of $\omega \Delta t(=0.22)<1$ and $k_{\|} v_{\| \text {cut }} \Delta t(=0.59)<1$, and it is much larger than $\Delta t$ used in simulation runs reported so far. The results here serve as an example that the numerical properties of the simulation plasma can indeed be improved with this scheme without sacrificing the physics.

Let us now compare the scheme with the one which keeps track of the fast particles at every time step. (This becomes necessary, for example, when collisions and/or the nonlinear parallel acceleration are important and we have to re-tag the particles.) Here, the ions and slow electrons are still treated the same way as before with Eqs. (10), (12a) and (12b), but the fast electrons are instead pushed using Eqs. (12b) and (13) and their adiabatic weights are given by Eq. (11). Since this is the linearized- $E_{\|}$scheme, Eq. (14) is used for the distribution. As stated earlier, the intrinsic numerical noise originates from the unity term in Eq. (14) as the result of 
the free-streaming motiun of $d x_{j}^{(0)} / d t=v_{\| j}^{(0)} \hat{b}$. Instead of neglecting the streaming motion for the fast electrons so that their contribution to the unity term becomes time independent, we can actually keep the motion intact while neglecting the time variation associated with the unity term in Eq. (14). The reason is that the zero-order trajectories for the homogeneously loaded fast particles give rise to the time invariant property

$$
\partial f^{(0)}\left(v_{\|}>v_{\| \text {cut }}\right) / \partial t=0
$$

i.e., the background distribution for the fast particles remains homogeneous and incompressible in the configuration space. In practice, we can either keep the time independent contribution of $f^{(0)}$ for the fast particles or neglect it totally when calculating the self-consisitent fields in the simulation. Although the time step has to be reduced in this scheme, the noise level still remains low. For $\theta=0.01, \tau=4, \nu_{\mathrm{ei}}=0$ and $\Delta t=2.18$, the time history for $\phi_{+}$based on this scheme of fast moving adiabatic electrons is shown in Fig. 10, where the real frequency is $\omega_{r}=0.045$ and the saturation amplitude is $\left|e \phi / T_{e}\right| \simeq 0.7 \%$. Except that the noise interference in the linear stage is slightly more pronounced, these results are very similar to those in Fig. 9, where Eq. (20) is used to account for the adiabatic electron response.

\subsection{Linearized- $E_{\|} \& \kappa$ Model}

Because the zerc-order trajectories for all the particles (ions, and slow and fast electrons) are given by Eqs. (12b) and (13) for the linearized- $E_{\|} \& \kappa$ algorithm, the time invariant property extends to all the particles and now becomes

$$
\frac{\partial f^{(0)}}{\partial i}=0
$$

and the unity term in Eq. (15) can then be neglected (or made constant) for all the particles. When that happens, the intrinsic numerical noise is totally eliminated from the simulation and an artificial initial noise has to be introduced instead. We have carried out a simulation using Eqs. (10) and (16) for the perturbed quantities for all the particles, i.e., without the adiabatic approximation, with the symmetry condition imposed on $\phi$, for the parameter values $\theta=0.01$, $\tau=4, v_{\mathrm{ei}}=0$, and $\Delta t=2.18$. To provide the initial noise for the simulation, we have kept the time variation of the $f^{(0)}$ contribution due to the ions. The results are shown in Fig. 11. As expected, the initial noise level is lower than those of Figs. 9 and 10 , because the electron free-streaming motion is completely eliminated. (For even lower initial noise, one can introduce an artificial perturbation instead of using the ion streaming noise.). The growth of the instability now spans nearly three decades, where $\omega=0.045+0.008 i$ and $\left|e \phi / T_{\mathrm{e}}\right|_{\mathrm{sat}} \simeq 0.6 \%$. The linear growth is nearly perfect. [Most remarkably, the linear as well as the nonlinear behavior of the instability are almost identical to those in Fig. 4 of Ref. 5, obtained from a Vlasov mode-coupling code, which solves for the distribution function $f$ in the $(k, q)$ space, i.e., the Fourier transformed $\left(\boldsymbol{x}, v_{\|}\right)$space.] Because of the presence of $\omega_{\mathrm{H}}$ oscillations, it may not be possible to increase the time step with the present scheme [10]. However, we can always use the algorithm prescribed by Eqs. (11), (12b), (13) and (17) for the fast electrons to eliminate the high frequency oscillations. We have carried out such a run with $\Delta t=2.18$ and the results are almost identical to the ones shown in Fig. 11, except for the nonlinear oscillation of $\left|\phi_{+}\right|$, which has a slightly higher 
frequency. To increase $\Delta t$, we again use Eq. (20) for the fast electrons, and use Eq. (15) only for the ions and the slow electrons. With the same parameters as those of Fig. 11 except for $\Delta t=5.45$, we again reproduce a nearly identical time history for $\phi_{+}$as shown in Fig. 12 . From the results in Figs. 9-12, we can also conclude that the slight deviations from the linear predictions of the measured frequencies and growth rates in the simulation are caused by the use of the normal mode approach to calculate the frequencies and growth rates, whereas the simulation solves an initial value problem.

\subsection{Comparisons and Discussion}

Let us now use the same scheme of direct substitution of the fast electron response, Eq. (20), into the gyrokinetic equation for the case in which the symmetry condition for $\phi$ is removed, i.e., $\phi(m, n) \neq \phi^{\prime \prime}(m,-n)$. Figure 13 gives the time evolution of $\phi_{ \pm}$for the linearized- $E_{\|}$run with $\tau=4, \theta=0.01, v_{\mathrm{ei}}=0, \Delta t=2.18$ and $v_{\| \mathrm{cut}}=0.3 v_{\mathrm{te}}$, where responses of the ions and slow electrons are calculated using Eqs. (10), (12a), (12b), and (14). The evolution is similar to that shown in Fig. 7, but with much reduced noise. It also confirms that the velocity space nonlinearity is indeed important for the saturation of the instability when $k_{\|}$is large. (When we used $\Delta t=5.45$ for the same simulation, a numerical instability was observed. The probable cause is $k_{\perp} V_{E \times B} \Delta t>1$ for some of the modes because of the large saturation amplitude.) The corresponding low noise/long-time-step linearized- $E_{\|} \& \kappa$ run again fails to saturate at the proper level with one mode dominating over the other. This type of behavior may be related to the streamers observed in fluid simulations [27].

While the above scheme, involving the direct substitution of the fast electron response into the gyrokinetic Poisson equation works well for increasing $\Delta t$ for the collisionless plasmas, the possibility of using the orbit averaging scheme[25] should be explored for the cases in which we are compelled to follow the fast particles. Besides collisions and parallel acceleration, the presence of magnetic shear and three dimensional geometries, which allow regions in which $k_{\|}=0$, may also make it necessary. This is because, when $k_{\|}$approaches zero, we may not be able to use $v_{\| \text {cut }}$ for the fast particles. However, $\omega_{\mathrm{H}}$ modes cease to exist in a sheared system[10] and the orbit averaging scheme is still applicable.

\section{Summary and Concluding Remarks}

In this paper, partially linearized particle simulation algorithms, in which the particles have time-varying weights were developed. These algorithms were applied to remove selected nonlinearities in the gyrokinetic simulation of drift waves in order to assess the importance of these nonlinearities as well as to suppress the intrinsic numerical noise and to increase the time step $\Delta t$ in the simulation. (The issue of large grid spacing will be discussed elsewhere.)

As we first reported in 1987[7] and as indicated by Figs. 1 and 2, the removal of the parallel acceleration nonlinearity was found not to have a significant qualitative effect on the gyrokinetic simulation results for drift waves [6] in the small $\theta\left(\equiv k_{||} / k_{y} \ll 1\right)$ cases. This can be understood on the basis of the arguments made in our previous work $[8,9]$. Briefly, the saturation takes place when the fluctuation level reaches a point such that the particle flux of the (linearly) nonresonant ions balances the resonant electron particle flux. Comparisons between Fig. 8 and Figs. 9-12 
also show that the low-noise/long-time-step partially linearized algorithms are also adequate for capturing the essential physics of the drift instability for larger $\theta$, in the case where the symmetry condition on $\phi$ is satisfied. Moreover, the agreement between the results of the particle code and the Vlasov mode-coupling code enables us to ascertain that the three-wave coupling due to the $\boldsymbol{E} \times \boldsymbol{B}$ advection is again the major reason for the nonlinear saturation in these cases. On the other hand, as shown in Figs. 6 and 7, when $\theta$ (or $\left.k_{\|}\right)$is large and the symmetry condition on $\phi$ is removed, the parallel acceleration nonlinearity does make difference in the saturation. There is, at present, no quantitatively correct theory for this case. At this point, the new algorithms have only been applied to simulations which produce semi-coherent states. Their application to more turbulent states in morc complicated geometries is needed.

For spatially (semi-) coherent states, the removal of the nonlinear term in the so-called multiple-scale model [10] used in the simulations of Lee et al. [5], Federici et al. [6], and Lee and Tang [17] was found to result in secular growth of the waves after the linear growth has saturated. This is because, unlike for codes which use the particle conserving multiple-scale term, the dominant modes of the density can grow without bound due to the coherent advection of $E \times B$-trapped groups of particles. However, the secular growth in the semi-coherent simulation can be eliminated, if the symmetry condition for the potential is imposed. Preliminary results of simulations which result in turbulent states indicate that the nonlinear term in the multiple-scale model has little effect on turbulent saturated states.

As we have mentioned earlier, the noise reduction method using partially-linearized algorithms is an extension of the technique originally used for the fully-linearized code [11]. It is different from the method used in a recent attempt to suppress the noise in a fully-nonlinear particle simulation by Kotchenreuther [13]. Both schemes have zero intrinsic noise, but their nonlinear evolution of the artificial noise may be different. For a partially (or fully) linearized system, the fluctuation-dissipation theorem $[1-3,10]$ is not applicable. The reason is that, in the absence of the parallel acceleration nonlinearity i.e., when $d v_{\|}^{(0)} / d t=0$ for all the particles, the simulation can never reach the thermal equilibrium. On the other hand, the fully nonlinear case should obey the fluctuation-dissipation theorem. Thus, the behavior of the noise in the "steady state" stages of the simulation may, therefore, be qiute different for the two methods. Furthermore, in order to ignore the zero-order contribution for the noise due to free streaming in the fully nonlinear simulation, one has to load the particles homogeneously in the whole phase space, which may become very cumbersome and wasteful at large velocities where the number of simulation particles that represent a physical particle becomes much larger than average. In our case, only the configuration space homo ${ }_{-}$eneity is needed. (The other requirement of phase space incompressibility is usually satisfied except for the particle conserving multi-scale model.) Nevertheless, the novel approach for the fully nonlinear case is very important and should be pursued further.

One interesting extension of the algorithm that uses time-varying weights is its usage for the fast-moving particles. By assuming their weights to be adiabatic, we can actually eliminate the high frequency oscillations from the simulation and, as we have demonstrated in the paper, even remove the restriction on $\Delta t$ imposed by the Courant condition. For electromagnetic simulation, this scheme is particularly useful for suppressing the numerical noise arising from the free-streaming motion of the fast electrons [23].

Another important application of the present algorithrns is the possibility of making mean- 
ingful comparisons of the results between the gyrokinetic particle code and fluid codes which include finite-Larmor-radius effects [28]. To account for the collisional dissipative effects in the particle code, we can use the Lorentz model for the electron-ion collisions $[21,22]$ and the newly developed gyrokinetic model for the ion-ion collisions [29].

\section{ACKNOWLEDGMENT}

This work was supported by U. S. DoE at Princeton Plasma Physics Laboratory through Contract No. DE-AC02-76CHO-3073 and partly (for A. M. D.) at the University of Maryland through Contract No. DEFG05-86ER53221. 


\section{References}

1. J. M. Dawson, Rev. Mod. Phys. 55, 403 (1983).

2. C. K. Birdsall and A. B. Langdon, Plasma Physics via Computer Simulation (McGraw-Hill Inc., 1985).

3. R. W. Hockney and Eastwood, J. W. Computer Simulation using Particles (McGraw-Hill Inc., 1981).

4. T. P. Armstrong, R. C. Harding, G. Knorr, and D. Montgomery, Methods of Computational Physics 9, 29-86 ( 1970).

5. W. W. Lee, J. A. Krommes, C. Oberman, ane R. A. Smith, Phys. Fluids 27, 2652 (1984).

6. J. F. Federici, W. W. Lee, and W. M. Tang, Phys. Fluids 30, 425 (1986).

7. A. M. Dimits and W. W. Lee, Partially Linearized Gyrokinetic Particle Simulation of Drift Instabilities, in Proceedings of the 12th Conference on the Numerical Simulation of Plasmas, paper-PW23 (Sarı Francisco, Califomia, 1987).

8. A. M. Dimits, Ph.D. thesis, Princeton University, 1988.

9. A. M. Dimits and W. W. Lee, Nonlinear Mechanisms for Drift Wave Saturation and Induced Particle Transport, Princeton University Plasma Physics Laboratory Report No. PPPL-2659 (Princeton, New Jersey, 1989), submitted to Phys. Fluids.

10. W. W. Lee, J. Comput. Phys. 72, 243 (1987).

11. J. A. Byers, Noise Suppression Techniques in Macroparticle Models of Collisionless Plasmas, in Proceedings of the Fourth Conference on Numerical Simulation of Plasmas (NRL, Washington DC 1970), p. 496.

12. J. P. Freidberg, R. L. Morse, and C. W. Nielson, Numerical Methods for Studying Linear Stability of Highly Inhomogeneous Plasmas, in Proceedings of the Third Annual Numerical Plasma Simulation Conference (Stanford University, CA, 1969).

13. M. Kotchenreuther, Bull. Am. Phys. Soc. 34, 2107 (1988).

14. B. I. Cohen, S. P. Auerbach, J. A. Byers, and H. Weitzner, Phys. Fluids 23, 2529 (1980).

15. W. W. Lee, Phys. Fluids 26, 556 (1983).

16. D. H. E. Dubin, J. A. Krommes, C. Oberman, and W. W. Lee, Phys. Fluids 26, 3524 (1983).

17. W. W. Lee and W. M. Tang, Phys. Fluids 31, 612 (1988).

18. R. D. Sydora, T. S. Hahm, W. W. Lee, and J. M. Dawson Phys. Rev. Lett. 64, 2015 (1990).

19. W. M. Tang, Nuclear Fusion 18, 1089 (1978).

20. A. Friedman, R. N. Sudan, and J. Denavit, J. Comput. Phys. 40, 1 (1980). 
21. R. Shanny, J. M. Dawson, and J. M. Greene, Phys. Fluids 10, 1281 (1967).

22. A. Boozer and G. Kuo-Petravic. Phys. Fluids 24, 851 (1981).

23. W. W. Lee, T. S. Hahm, and J. V. W. Reynders, Considerations of Electron Dynamics in a Gyrokinetic Plasma, Sherwood Theory Conference, paper-3D2 (1989)

24. J. V. W. Reynders and W. W. Lee, private communication.

25. B. I. Cohen, T. A. Brengle, D. B. Conley, and R. P. Freis, J. Comput. Phys. 38, 45 (1980).

26. A. M. Dimits, Phys. Fluids, (1990), to appear.

27. J. F. Drake; P. N. Guzdar, and A. B. Hassam, Phys. Rev, Lett. 61, 2205 (1988).

28. A. M. Dimits, J. F. Drake, and W. W. Lee, Kinetic Effects on Ion Temperature Gradient Driven Turbulence, Sherwood Theory Conference, paper-3D27 (1990).

29. X. Q. Xu and M. N. Rosenbluth, Numerical Simulation of Ion Temperature Gradient Driven Modes in the Presence of Ion-Ion Collisions, submitted to Phys. Fluids (1990). 


\section{Figures}

Fig. 1. Time Evolution of $\phi(1,1)$ and $\phi(1,-1)$, denoted by $\phi_{ \pm}$, for the drift instability using the nonlinear gyrokinetic code with $\theta=0.002, \tau=100, \nu_{\text {ei }}=0.001, \Delta t=1.09$, and $\left(k_{x}, k_{y}\right)=0.842(m, n)$.

Fig. 2. Time e', viution of $\phi_{ \pm}$using the linearized- $E_{\|}$algorithm of Sec. 5.1 for $\theta=0.002$, $\tau=100, \nu_{\mathrm{el}}=0.001$, and $\Delta t=1.09$.

Fig. 3. Time history for the electron particle flux induced by the in tability showin in Fig. 2.

Fig. 4. Electron particle flux calculated from a linearized- $E_{\|}$code with non-self-consistent electric potentials similar to those of Fig. 1.

Fig. 5. Diagram showing the decrease of the total (weighted) number of the electrons in the resonance region.

Fig. 6. Time evolution of $\phi_{ \pm}$using the fully nonlinear gyrokinetic code with $\theta=0.01, \tau=4$, $\nu_{\mathrm{ei}}=0$, and $\Delta t=2.18$.

Fig. 7. Time evolution of $\phi_{ \pm}$using the linearized- $E_{\|}$algorithm of Sec. 5.1 with $\theta=0.01, \tau=4$, $\nu_{\mathrm{ei}}=0$, and $\Delta t=2.18$.

Fig. 8. Time history of $\phi_{+}$using the nonlinear gyrokinetic code with $\phi_{+}=\phi_{-}^{*}, \theta=0.01, \tau=4$, $\nu_{\mathrm{ei}}=0$, and $\Delta t=2.18$.

Fig. 9. Time history of $\phi_{+}$using the low-noise/long-time-step linearized- $E_{\|}$algorithm with the same parameters as those used in Fig. 8 except for $\Delta t=5.45$.

Fig. 10. Time history of $\phi_{+}$using the low-noise linearized- $E_{\|}$algorithm including the adiabatic approximation for the fast electrons with the same parameters as those used in Fig. 8.

Fig. 11. Time history of $\phi_{+}$using the low-noise linearized- $E_{\|} \& \kappa$ algorithm with the same parameters as those used in Fig. 8.

Fig. 12. Time history of $\phi_{+}$using the low-noise/long-time-step linearized- $E_{\|} \& \kappa$ algorithm with the same parameters as those used in Fig. 8 except for $\Delta t=5.45$.

Fig. 13. Time history of $\phi_{ \pm}$using the low-noise/long-time-step linearized- $E_{\|}$algorithm with the same parameters as those used in Fig. 7. The smalier time step used here is necessary due to the large saturation amplitude. 

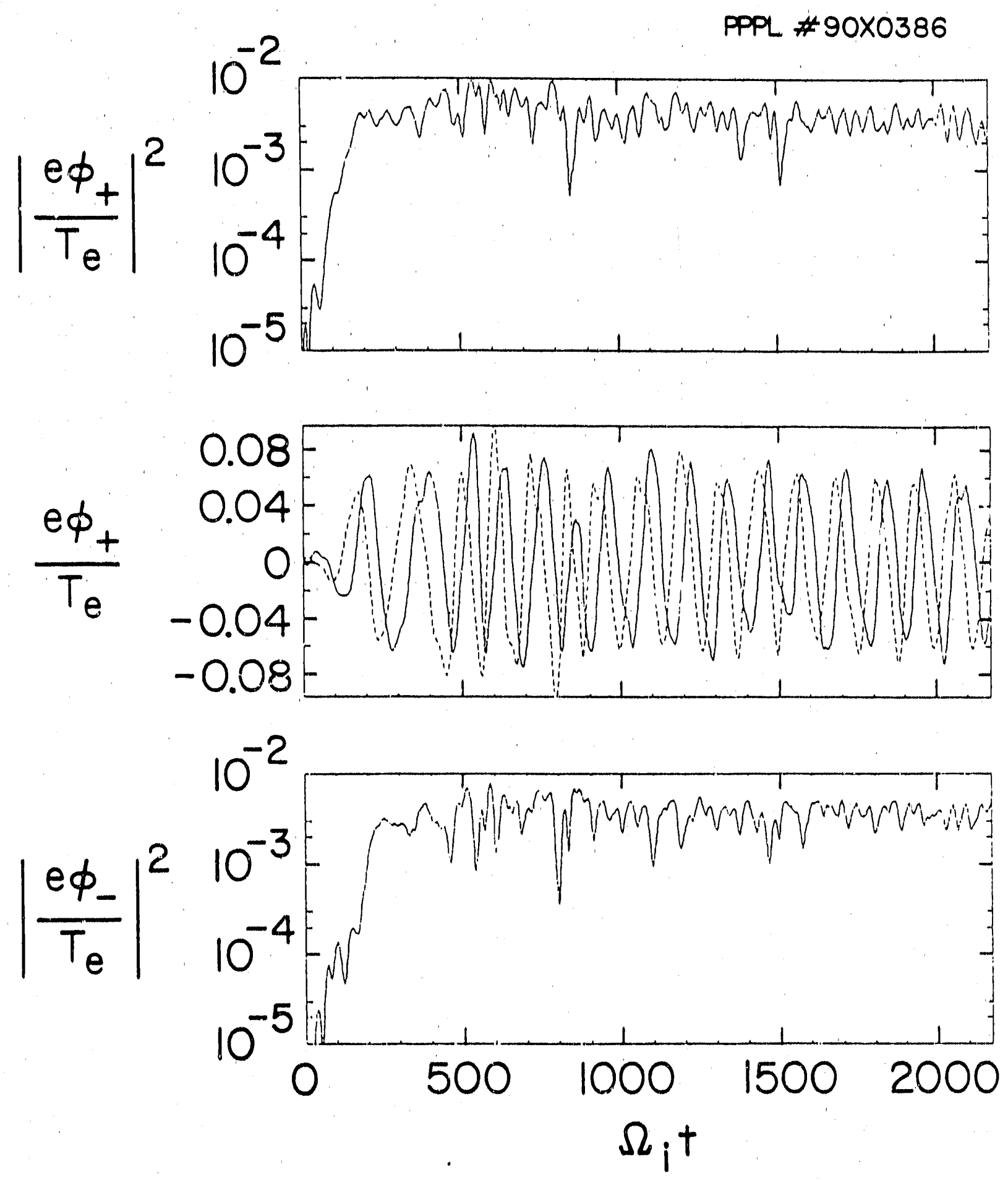

F1g. 1 
PPPL \#90X0387
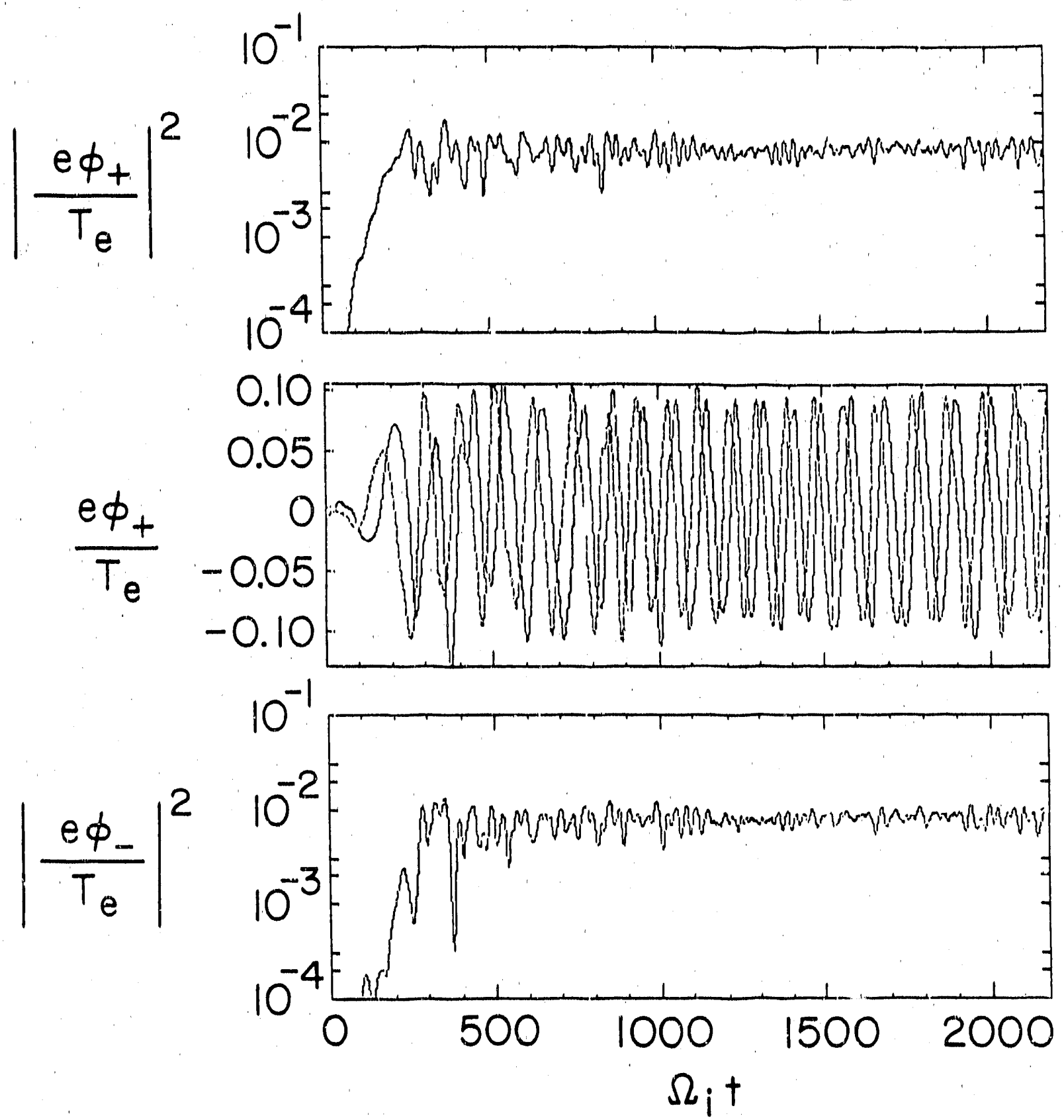

F1g. 2 


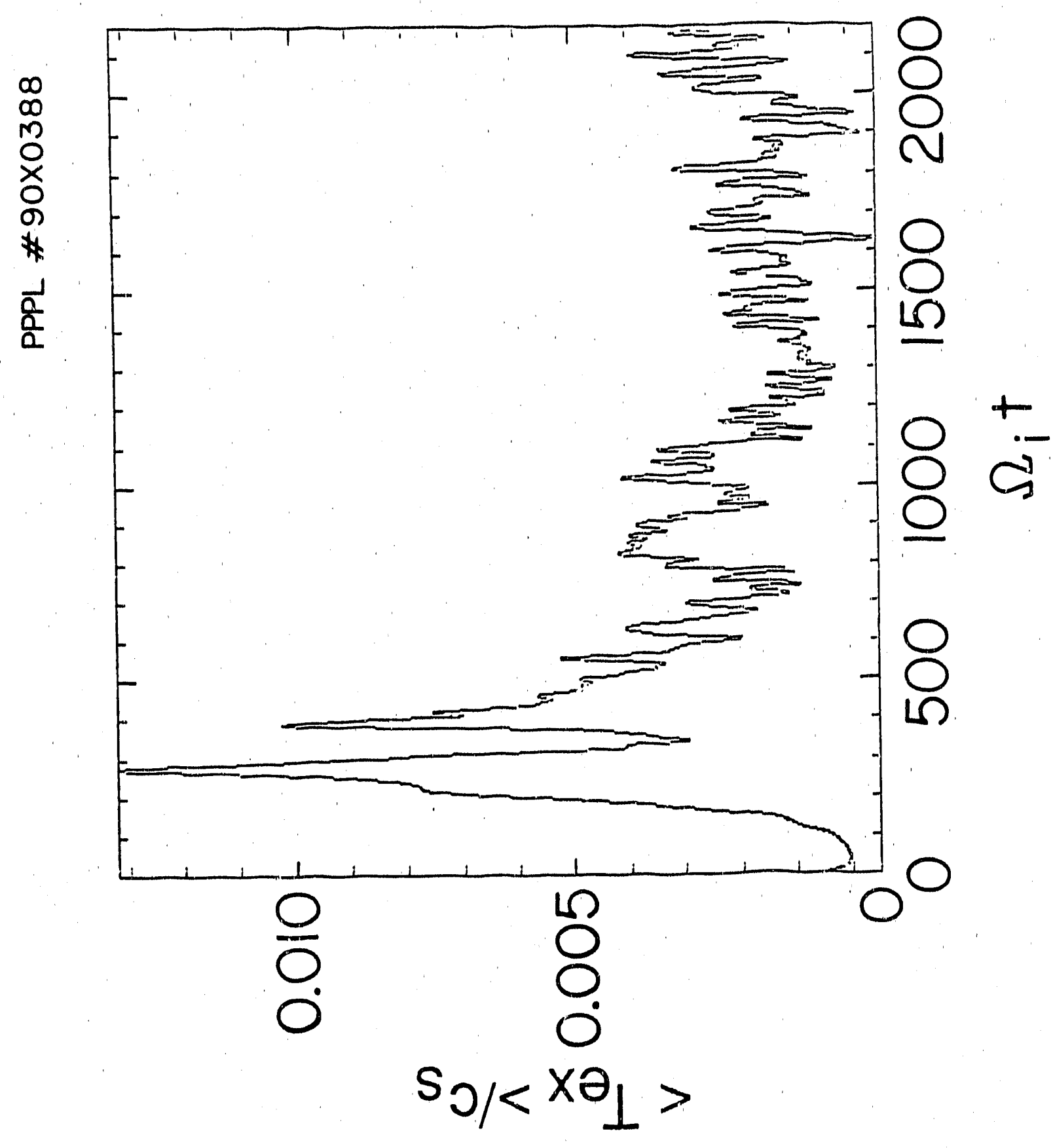




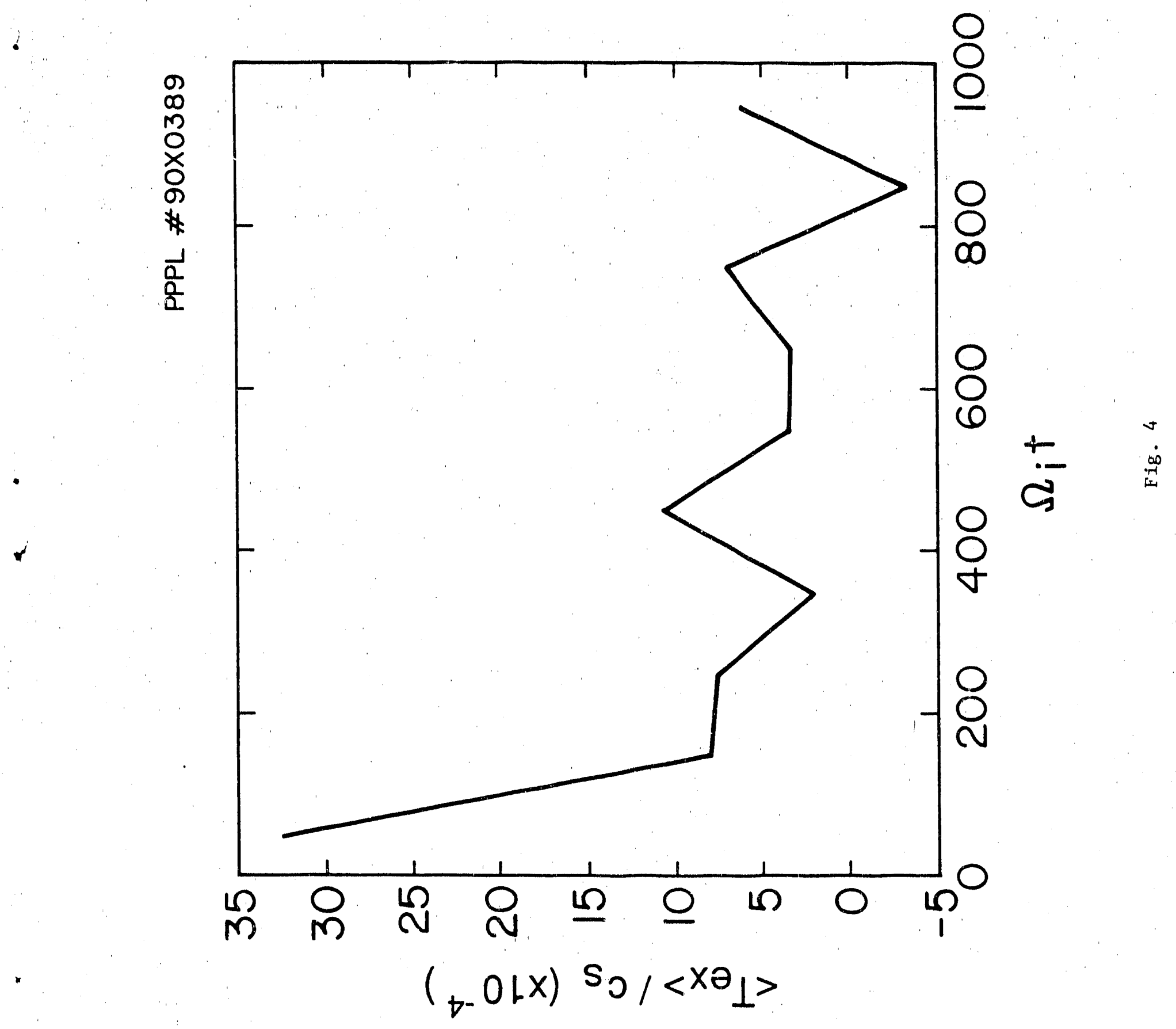




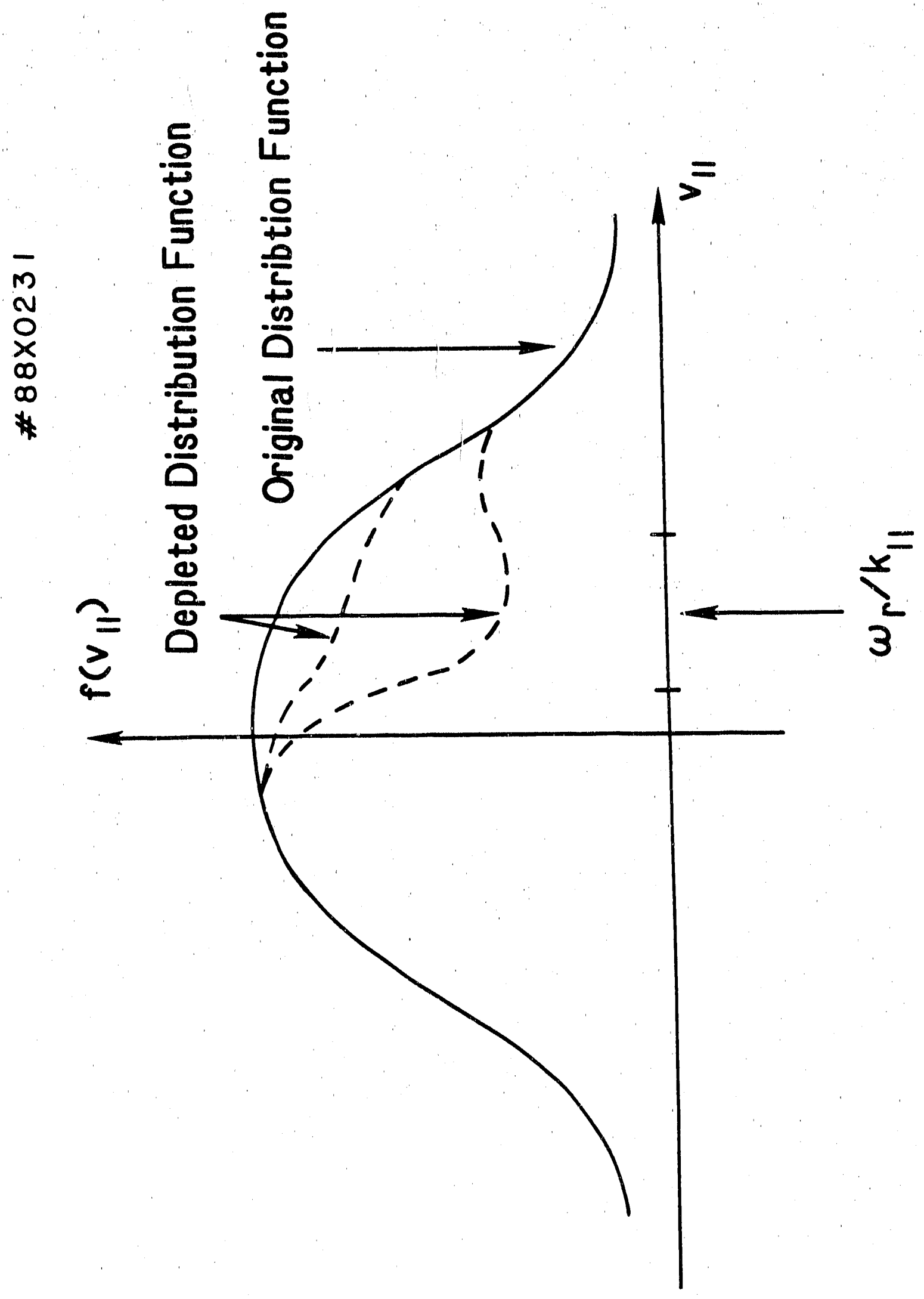

in 

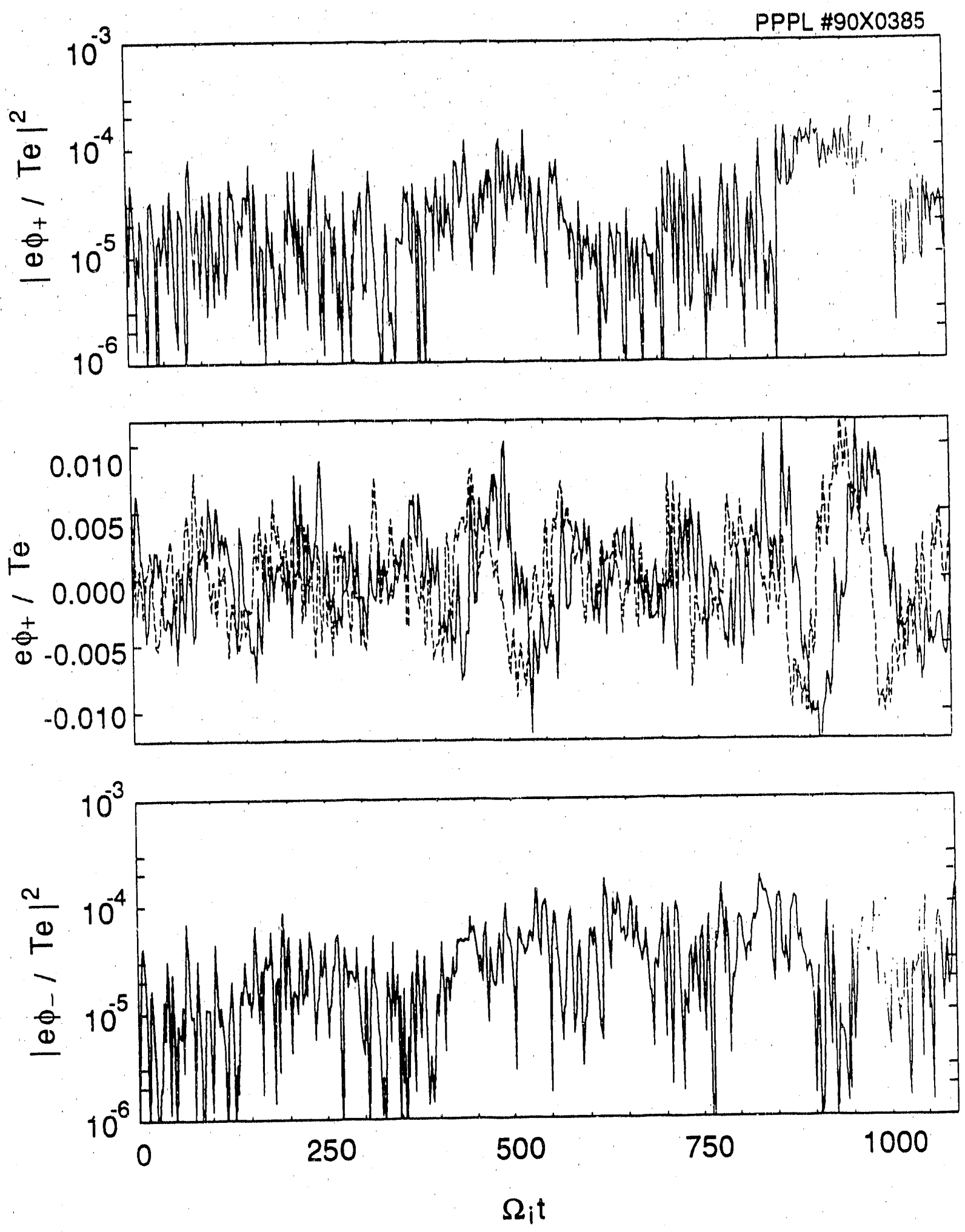

FIg. 6 

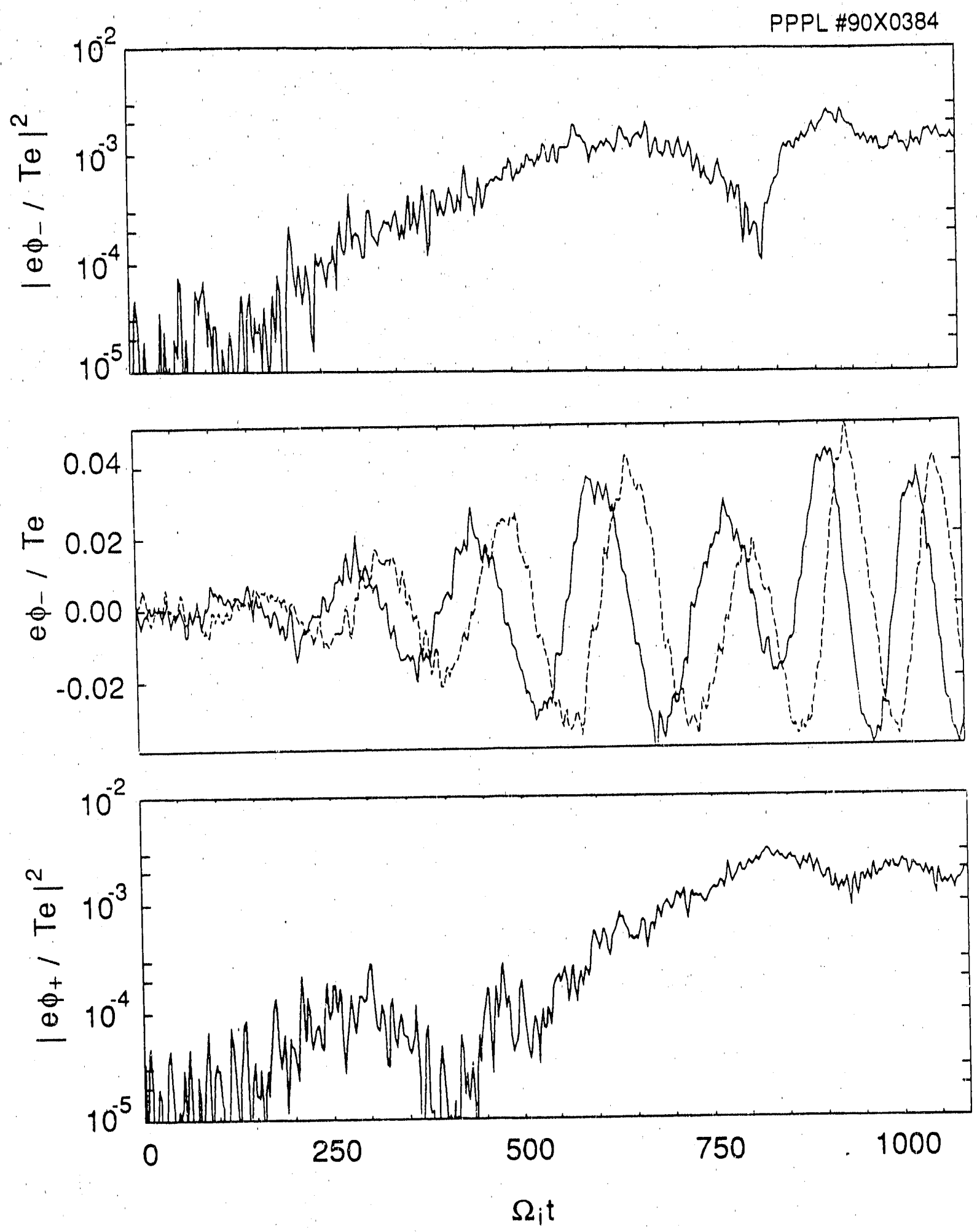

Fig. 7 

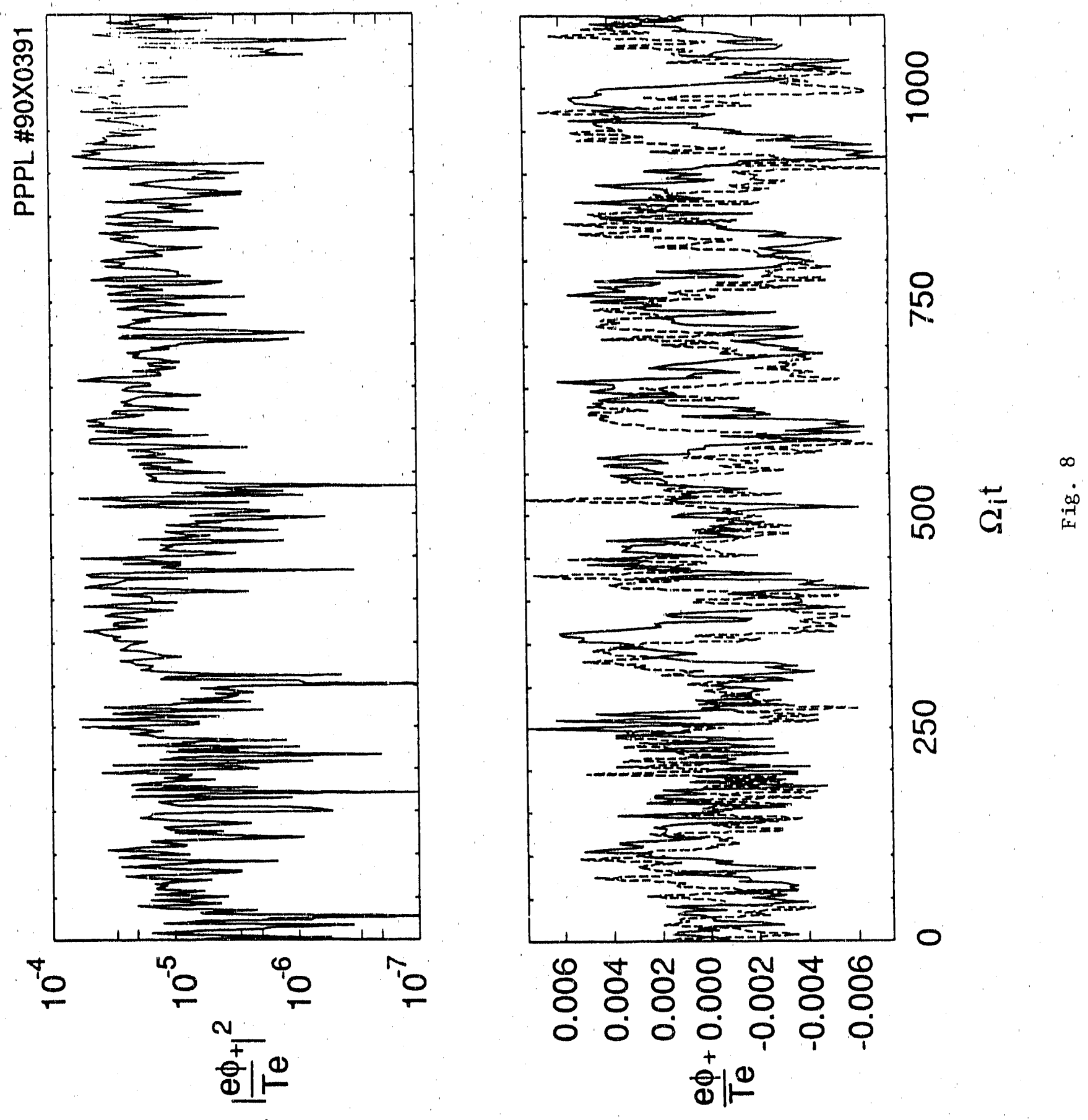

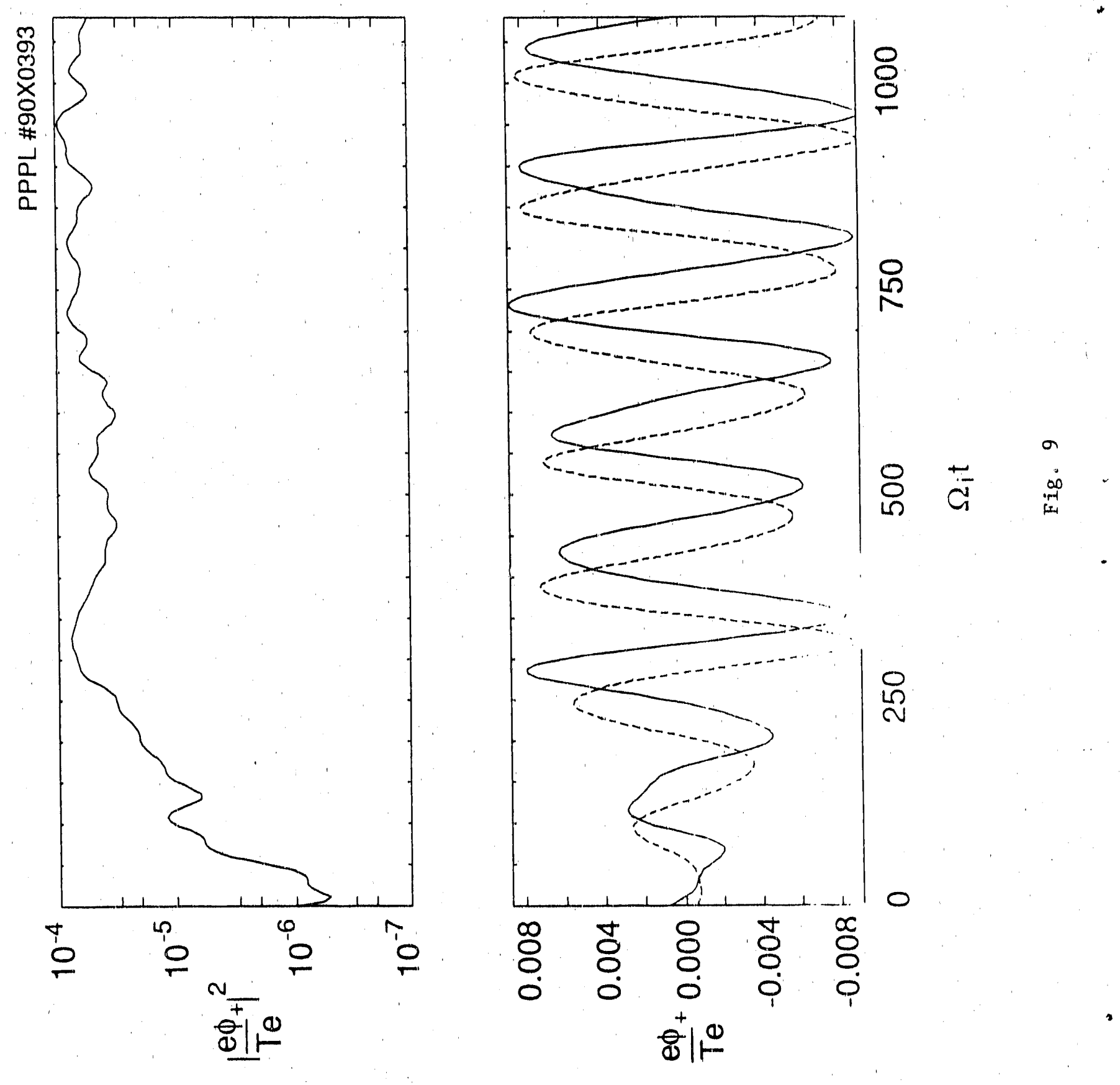

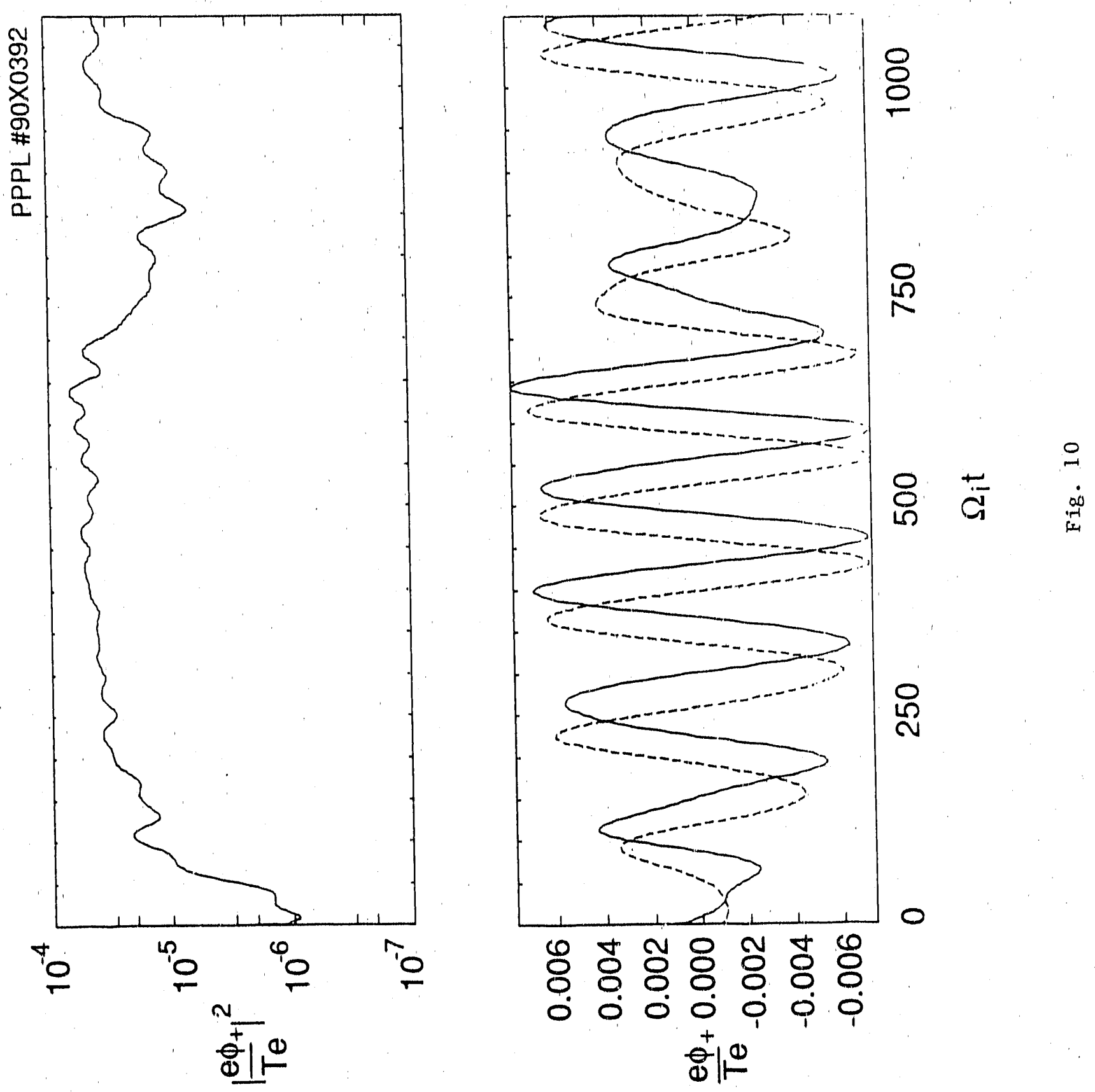

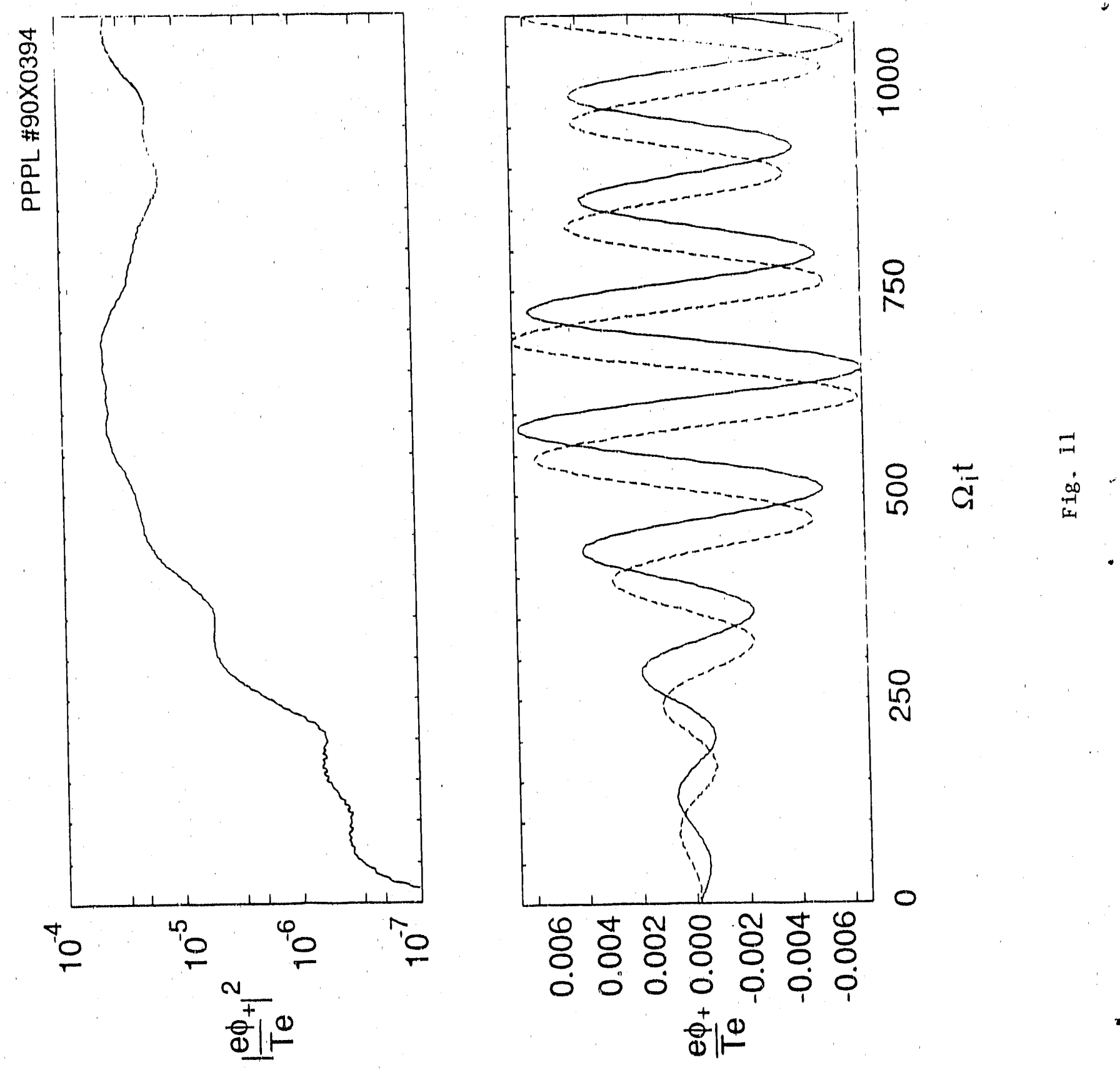

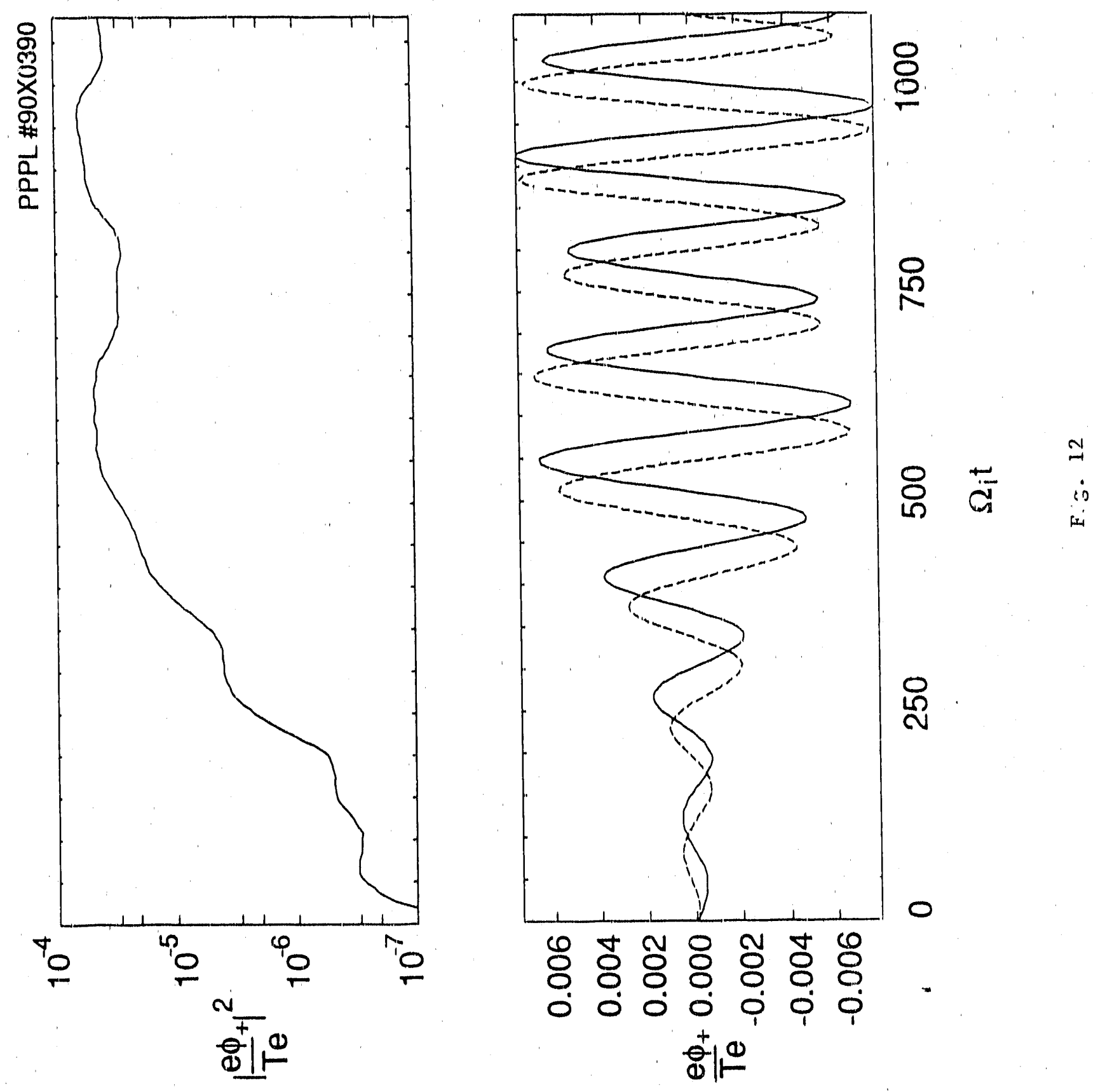

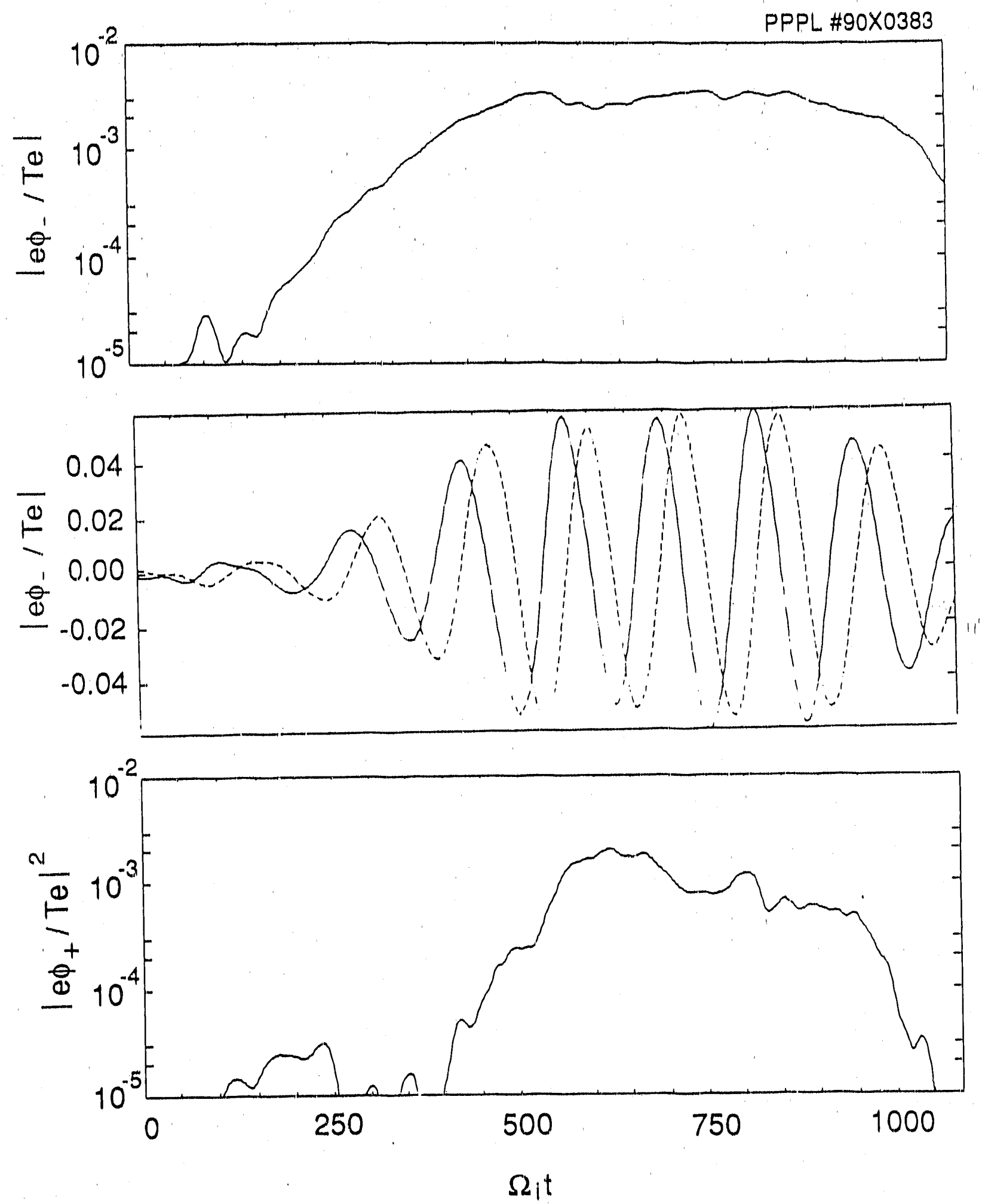

F18. is 

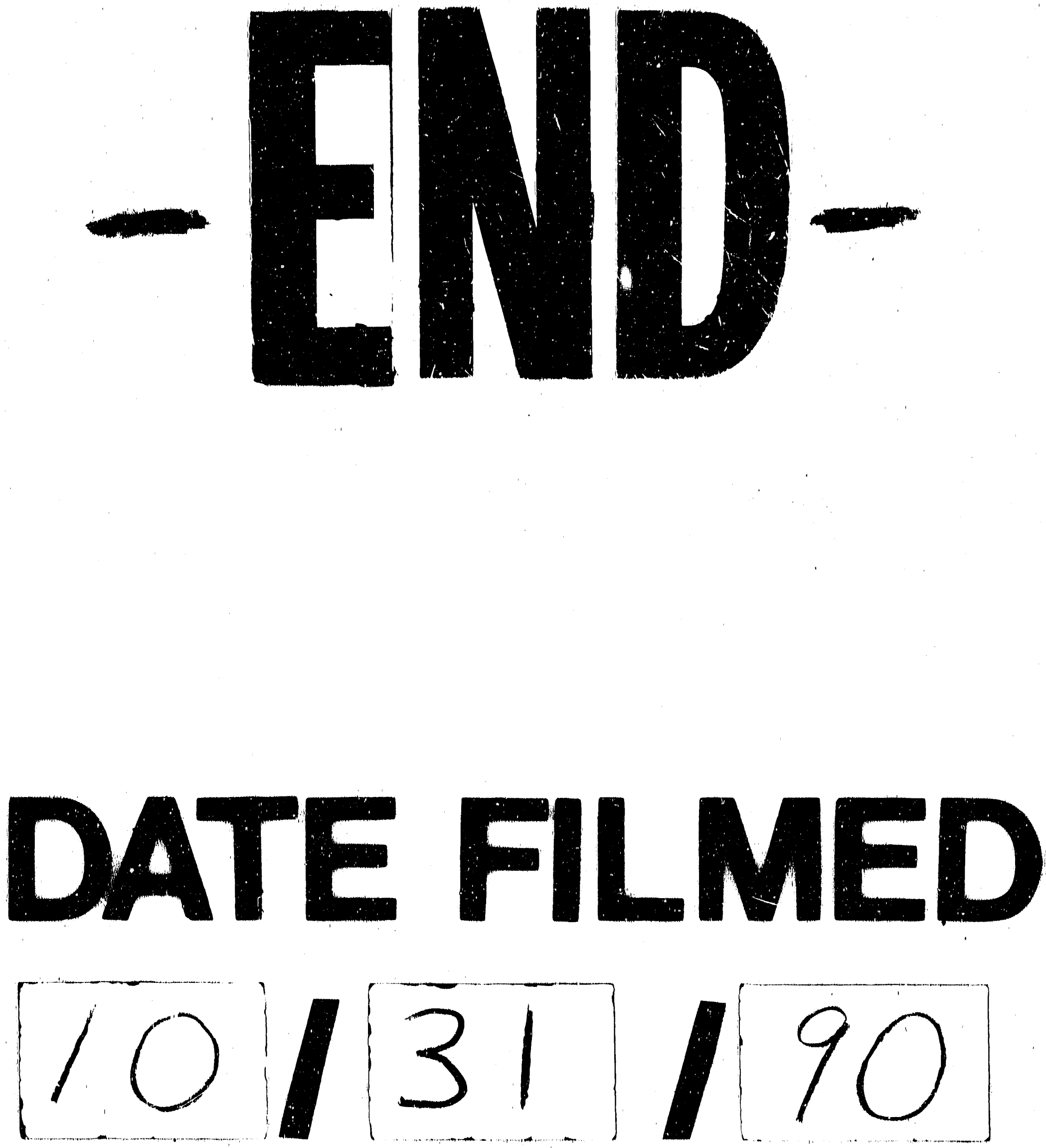
